\title{
Effects of the local resonance in bending on the longitudinal vibrations of reticulated beams ${ }^{\text {th }}$
}

\author{
C. Chesnais ${ }^{* a}$, C. Boutin ${ }^{\mathrm{b}}, \mathrm{S}$. Hans $^{\mathrm{b}}$ \\ ${ }^{a}$ Université Paris-Est, IFSTTAR, GERS, 14-20 Boulevard Newton, \\ F-77447 Champs-sur-Marne, Marne-la-Vallée Cedex 2, France \\ ${ }^{b}$ Université de Lyon, LGCB/LTDS CNRS 5513, ENTPE, \\ 3 rue Maurice Audin, F-69518 Vaulx-en-Velin Cedex, France
}

\begin{abstract}
This work investigates the dynamic behaviour of reticulated beams obtained by repeating a unit cell made up of interconnected beams or plates forming an unbraced frame. As beams are much stiffer in tension-compression than in bending, the longitudinal modes of such structures (governed by tensioncompression at the macroscopic scale) can appear in the same frequency range as the bending modes of the elements. The condition of scale separation being respected for compression, the homogenization method of periodic discrete media is used to rigorously derive the macroscopic behaviour at the leading order. In the absence of bending resonance, the longitudinal vibrations of the structure are described at the macroscopic scale by the usual equation for beams in tension-compression. When there is resonance, the form of the equation is unchanged but the real mass of the structure is replaced by an effective mass which depends on the frequency. This induces an abnormal response in the neighbourhood of the natural frequencies of the resonating elements. This paper focuses on the consequences on the modal properties and the transfer function of the reticulated structure. The same macroscopic mode shape can be associated with several natural frequencies
\end{abstract}

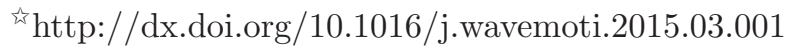

(c) 2015. This manuscript version is made available under the CC-BY-NC-ND 4.0 license http://creativecommons.org/licenses/by-nc-nd/4.0/

*Corresponding author. Tel: 0033181668378

Email addresses: celine.chesnais@ifsttar.fr (C. Chesnais), claude.boutin@entpe.fr (C. Boutin), stephane.hans@entpe.fr (S. Hans) 
of the structure (but the deformation of the elements at the local scale is different). Moreover the vibrations are not transmitted when the effective mass is negative. These phenomena are first evidenced theoretically and then illustrated with numerical simulations.

Key words: Local resonance, effective mass, reticulated material, metamaterial, homogenization

\section{Introduction}

Locally resonant materials or metamaterials are a class of composite materials with a high stiffness contrast between the constituents. The propagation of waves in the stiff component with a wavelength much greater than the heterogeneity size can then induce the resonance of the soft component. This phenomenon which differs from diffraction leads to unusual effective properties investigated in the pioneering work of Auriault and Bonnet in 1985 [1] (see also [2]) and observed experimentally in [3] and [4]. In particular, the effective density is different from the real density and depends on the frequency. The description of such composites at the macroscopic scale is a generalization of the Newtonian mechanics. This question is frequently addressed with mass-spring models (such as the Maxwell-Rayleigh model cited in [5]) which are difficult to realize in practice. The stratified composite studied by Auriault and Bonnet and the reticulated structure considered in this paper are more realistic systems.

Indeed, in $[6,7]$, it was shown that reticulated materials with only one constituent can also behave as locally resonant materials. In that case, the stiffness contrast comes from the geometry of the microstructure. Reticulated materials are made up of interconnected beams or plates. Examples include materials of millimetric size such as foams, plants, bones, of metric size such as the sandwich panels, stiffened plates and truss beams used in aerospace and marine structures, of decametric size such as buildings. Since beams and plates are much stiffer in tension-compression than in bending, the propagation of compressional waves with a long wavelength and the local bending modes of the elements can occur in the same frequency range. The local resonance in bending of a reticulated material is used in [8] to attenuate vibrations over desired frequency ranges.

In this paper, we investigate the consequences of the local resonance in bending on the dynamic behaviour of periodic frame structures. Instead of 
considering wave propagation as in $[6,7]$, emphasis is put on the modification of the features of the longitudinal modes. For the first modes of a structure with a sufficiently large number of periods (or cells), deformations occur on a length scale much greater than the size of a period. Therefore the homogenization method of periodic discrete media (HPDM method) can be used to obtain a macroscopic description. This method, elaborated by Caillerie [9] has been extended by a systematic use of scaling based on dimensional analysis $[10,11]$ and applied to situations with local resonance [6, 7]. Its main advantages are that the macroscopic behaviour is derived rigorously from the properties of the basic frame and that it provides an analytic formulation which enables to understand the role of each parameter. This method has already given interesting results on the transverse dynamics of frame structures [11].

The framework of the study is described in Section 2 and the details of the HPDM method are given in Appendix B. Section 3 presents the two possible macroscopic behaviours: without and with local resonance. In Section 4, the consequences of the local resonance on the free and forced vibrations are analysed. These results are confirmed by finite element simulations. Finally, Section 5 discusses the potential applications of this work. The differences between the idealized reticulated structures and real buildings are examined and the important points for the design of new structures with prescribed properties are highlighted. Note that the demonstrations of some results about the harmonic vibration of Euler-Bernoulli beams used in this article are gathered in Appendix A.

\section{Framework of the study}

\subsection{Studied structures and kinematic descriptors}

The studied structures are constituted by a pile of a large number $N$ of identical unbraced frames called cells and made of a floor supported by two walls (see Figure 1). The walls and the floors are beams or plates which behave as Euler-Bernoulli beams in out-of-plane motion. They are linked by perfectly stiff and massless nodes. The characteristics of the floors $(j=f)$ and the walls $(j=w)$ are: $\ell_{j}$ length, $a_{j}$ thickness, $h$ depth in the direction

$\mathbf{e}_{3}, A_{j}=a_{j} h$ cross-section area, $I_{j}=a_{j}^{3} h / 12$ second moment of area in the direction $\mathbf{e}_{3}, \rho_{j}$ density, $E_{j}$ elastic modulus.

This paper deals with the harmonic vibrations of the structure at the unknown circular frequency $\omega$ of the longitudinal modes. Therefore, every 
(a)

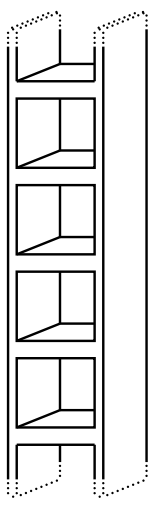



(b)

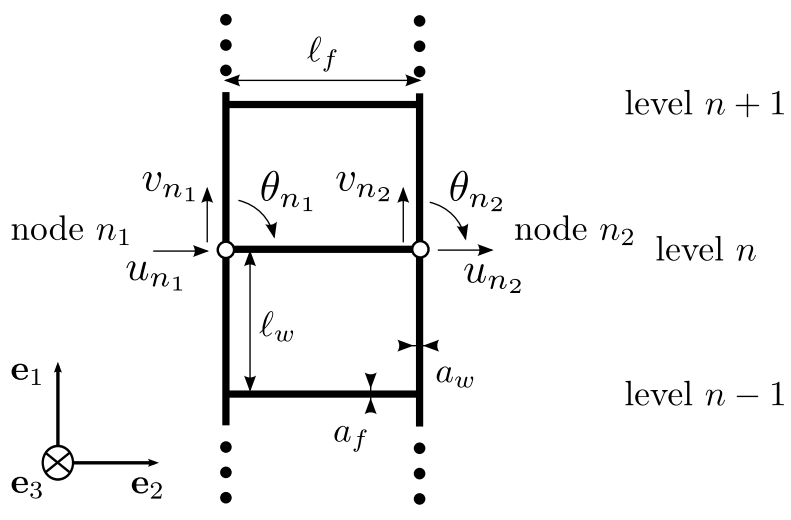

Figure 1: (a) Examples of studied structures; (b) notation.

variable can be written in the following way: $X(t)=\Re\left(X \mathrm{e}^{\mathrm{i} \omega t}\right)$ where $t$ is the time. Since the study is conducted within the framework of the small strain theory and the linear elasticity, the time dependence can be simplified and will be systematically omitted.

As explained in Appendix B, the HPDM method begins with the discretization of the dynamic balance. The study of the momentum balance of the whole structure is exactly replaced by the study of the momentum balance of the nodes. Since the process is performed without loss of information, it is possible to use the motions of the nodes as kinematic descriptors of the motions of the structure. Figure 1 shows that each level $n$ contains two nodes: $n_{1}$ on the left and $n_{2}$ on the right. Their motion in the plane $\left(\mathbf{e}_{1}, \mathbf{e}_{2}\right)$ is described by three kinematic variables: $v_{n_{j}}$ for the displacement in the direction $\mathbf{e}_{1}, u_{n_{j}}$ for the displacement in the direction $\mathbf{e}_{2}$ and $\theta_{n_{j}}$ for the rotation with $j=1$ or 2 .

Because of the longitudinal symmetry of the structures, it is possible to uncouple the transverse and longitudinal kinematics thanks to a change of variables. The six variables describing the motions of the two nodes of the level $n$ can be replaced by (see Figure 2): 
(a)

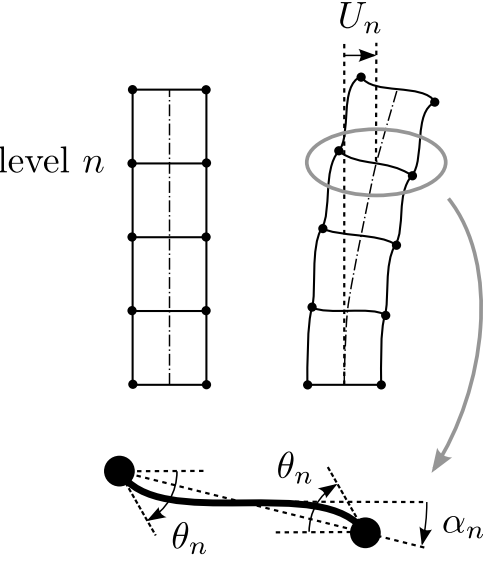

(b)

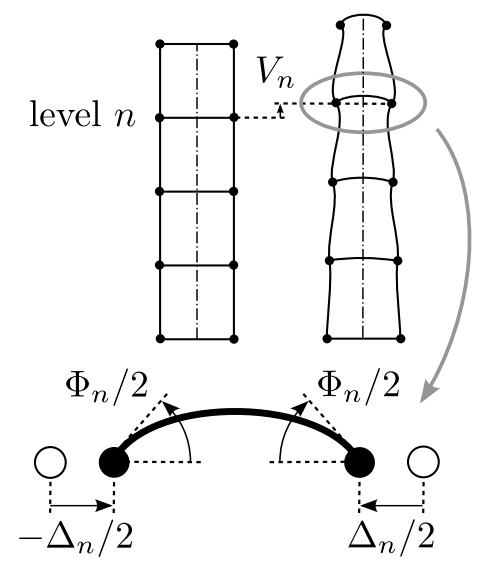

Figure 2: Variables of the (a) transverse and (b) longitudinal kinematics

- three variables associated to the rigid body motion of the level $n$ :

$$
\begin{aligned}
U_{n} & =\frac{u_{n_{1}}+u_{n_{2}}}{2} & \text { mean transverse displacement } \\
V_{n} & =\frac{v_{n_{1}}+v_{n_{2}}}{2} & \text { mean longitudinal displacement } \\
\alpha_{n} & =\frac{v_{n_{1}}-v_{n_{2}}}{\ell_{f}} & \text { global rotation }
\end{aligned}
$$

- three variables corresponding to its deformation:

$$
\begin{aligned}
\theta_{n} & =\frac{\theta_{n_{1}}+\theta_{n_{2}}}{2} & & \text { mean rotation of the nodes } \\
\Phi_{n} & =\theta_{n_{2}}-\theta_{n_{1}} & & \text { differential rotation of the nodes } \\
\Delta_{n} & =u_{n_{2}}-u_{n_{1}} & & \text { transverse dilatation }
\end{aligned}
$$

The transverse kinematics is governed by $(U, \alpha, \theta)$ and the longitudinal kinematics by $(V, \Phi, \Delta)$. This paper focuses on the longitudinal vibrations. The study of the transverse vibrations can be found in $[10,11]$.

Then, the discrete description is transformed into a continuous beam description thanks to the principles of homogenization (Appendix B). The 
key assumption is that the cell size in the direction of periodicity $\ell_{w}$ is small compared to the characteristic size $L$ of the vibrations of the structure. This condition of scale separation implies that the method is limited to the first modes of vibration which have wavelengths that are much longer than the cell size. The existence of a macroscopic scale is expressed by means of the macroscopic space variable $x$ in the direction of periodicity $\mathbf{e}_{1}$. The kinematic descriptors are considered as the discrete values of continuous functions of the space variable $x$, e.g. $V_{n}=V_{\epsilon}\left(x_{n}=n \ell_{w}\right)$. These functions are assumed to converge when the scale ratio $\epsilon=\ell_{w} / L$ approaches zero and they are expanded in powers of $\epsilon$ :

$$
V_{\epsilon}(x)=V^{0}(x)+\epsilon V^{1}(x)+\epsilon^{2} V^{2}(x)+\ldots
$$

where $V^{j}(x)$ is a continuous function of order $j$. Afterwards, the physically observable motion of a given order in $\epsilon$ will be written with a tilde: $\tilde{V}^{j}(x)=\epsilon^{j} V^{j}(x)$. The exact motion of the level is $V_{n}$ whereas the function $V^{0}(x)$ is the approximation at the leading order which appears in the macroscopic description. In the sequel, we will only study the leading order of the descriptors unless it is explicitly specified. Similarly, all other unknowns, including the natural frequency of the structure, are expanded in powers of the scale ratio $\epsilon$.

\subsection{Local resonance and normalization}

The aim of this paper is to investigate the consequences of the local resonance in bending of the floors on the longitudinal vibrations of the structure. Therefore, we have to design a structure such as the two phenomena occur simultaneously. The frequencies of the longitudinal modes depend essentially on the properties of the walls whereas the frequency range of the local resonance depends on the properties of the floors. This physics is introduced in the HPDM method thanks to the normalization which consists in scaling all the parameters according to the powers of $\epsilon$ (Appendix B).

The natural frequencies of the structure are close to the ones of the walls considered as clamped-free beams [Eq. (27) of Appendix A]. Since the walls vibrate at the macroscopic scale characterized by the length $L$, the order of magnitude of the frequency range of interest is:

$$
\omega=O\left(\frac{1}{L} \sqrt{\frac{E_{w}}{\rho_{w}}}\right)
$$


The floors are in resonance if their bending wavelength $\lambda_{b f}$ at the studied frequency has the same order of magnitude as $\ell_{f}$. According to Eq. (28) of Appendix A, this means that:

$$
\beta_{f} \ell_{f}=2 \pi \frac{\ell_{f}}{\lambda_{b f}}=\ell_{f} \sqrt[4]{\frac{\rho_{f} A_{f} \omega^{2}}{E_{f} I_{f}}}=O(1)
$$

If we assume that the walls and the floors are made of the same material and that $\ell_{f}=O\left(\ell_{w}\right)$, the introduction of Eq. (4) leads to the following condition:

$$
O\left(\frac{\ell_{w}^{4}}{a_{f}^{2} L^{2}}\right)=O(1) \quad \Rightarrow \quad \frac{a_{f}}{\ell_{w}}=O(\epsilon)
$$

In Section 3, we will compare two situations. In the first case, the floors are thick and they have a quasi-static behaviour at the frequencies of the longitudinal modes of the structure $\left(\beta_{f} \ell_{f}<<1\right)$. The chosen normalization is:

$$
\frac{a_{w}}{\ell_{w}}=O(\sqrt{\epsilon}) \quad ; \quad \frac{a_{f}}{\ell_{w}}=O(\sqrt{\epsilon}) \quad ; \quad \frac{\ell_{f}}{\ell_{w}}=O(1) \quad ; \quad \tilde{\omega}_{0}=O\left(\frac{1}{L} \sqrt{\frac{E_{w}}{\rho_{w}}}\right)
$$

where $\tilde{\omega}_{0}$ is the leading order of the circular frequency of the longitudinal vibrations [Eq. (46) of Appendix B]. The order of magnitude of the thickness to length ratios is $\sqrt{\epsilon}$ because it must remain small enough for the elements to be modelled by Euler-Bernoulli beams.

In the second case, $\beta_{f} \ell_{f}=O(1)$ and the floors are in resonance. The corresponding normalization is:

$$
\frac{a_{w}}{\ell_{w}}=O(\sqrt{\epsilon}) \quad ; \quad \frac{a_{f}}{\ell_{w}}=O(\epsilon) \quad ; \quad \frac{\ell_{f}}{\ell_{w}}=O(1) \quad ; \quad \tilde{\omega}_{0}=O\left(\frac{1}{L} \sqrt{\frac{E_{w}}{\rho_{w}}}\right)
$$

The thickness to length ratio of the walls is unchanged in order to avoid the resonance of the whole cell, which leads to a more complex behaviour [12]. As a result, the order of magnitude of $a_{f} / a_{w}$ is not a whole power of $\epsilon$ and the unknowns must now be expanded in powers of $\sqrt{\epsilon}$. For example, the mean longitudinal displacement becomes:

$$
V_{\epsilon}(x)=V^{0}(x)+\epsilon^{1 / 2} V^{1 / 2}(x)+\epsilon V^{1}(x)+\epsilon^{3 / 2} V^{3 / 2}(x)+\epsilon^{2} V^{2}(x)+\ldots
$$




\section{Macroscopic descriptions}

As explained in Appendix B, the study of the longitudinal vibrations of a structure as in Figure 1 with the HPDM method provides three balance equations [Eq. (44)]:

- $(\mathrm{SN})$ is the balance of the vertical forces,

- (DT) is the balance of the differential horizontal forces,

- (DM) is the balance of the differential moments.

At the leading order, (SN) depends only on the mean vertical displacement $V$ and it corresponds to the differential equation of the equivalent beam. The two other equations describe the inner equilibrium of the frame. They serve to determine the "hidden" variables $\Delta, \Phi$ and to study the deformation of the elements at the local scale.

\subsection{Quasi-static state at the local scale}

We first present the macroscopic beam equivalent to a structure with thick enough elements to have a quasi-static state at the local scale at the frequencies of the longitudinal modes of the structure. The corresponding normalization is given in Eq. (7) but a systematic study in which the properties of the elements vary [10] shows that the longitudinal vibrations with a quasi-static state at the local scale are always described by the same equation. In that case, we obtain at the leading order the classical description of a beam in tension-compression with the compression modulus of the two walls $2 E_{w} A_{w}$ and the linear density $\Lambda$.

$$
\begin{gathered}
(\mathrm{SN} 0) \quad \Rightarrow \quad \Lambda \tilde{\omega}_{0}^{2} \tilde{V}^{0}(x)+2 E_{w} A_{w} \tilde{V}^{0 \prime \prime}(x)=0 \\
\text { with } \quad \Lambda=\Lambda_{w}+\Lambda_{f} \quad ; \quad \Lambda_{w}=2 \rho_{w} A_{w} \quad ; \quad \Lambda_{f}=\rho_{f} A_{f} \frac{\ell_{f}}{\ell_{w}}
\end{gathered}
$$

Once the mean vertical displacement $V$ is known, the transverse dilatation $\Delta$ and the differential rotation of the nodes $\Phi$ are deduced from the two remaining balance equations:

$$
\begin{aligned}
& (\mathrm{DT}) \quad \Rightarrow \quad \frac{12 E_{f} A_{f}}{\ell_{w} \ell_{f}} \tilde{\Delta}^{3}(x)+3 K_{w} \tilde{\Phi}^{0 \prime}(x)=0 \\
& (\mathrm{DM}) \quad \Rightarrow \quad\left(K_{f}+3 K_{w}\right) \tilde{\Phi}^{0}(x)-\Lambda_{f} \ell_{f} \tilde{\omega}_{0}^{2} \tilde{V}^{0}(x)=0 \\
& \text { with } \quad K_{w}=\frac{24 E_{w} I_{w}}{\ell_{w}^{2}} \quad \text { and } \quad K_{f}=\frac{12 E_{f} I_{f}}{\ell_{w} \ell_{f}}
\end{aligned}
$$


Note that $\tilde{\Phi}^{0}(x)$ and $\tilde{\Delta}^{3}(x)$ depend on the stiffness of the elements in bending $K_{w}$ and $K_{f}$.

\subsection{Local resonance}

We now investigate the effects of the bending resonance by considering a structure with thinner floors and corresponding to the normalization of Eq. (8). Because of the thickness contrast between the walls and the floors, $\Lambda_{f} / \Lambda_{w}=O(\sqrt{\epsilon})$ and $\Lambda_{f}$ is negligible compared to $\Lambda_{w}$. The inertial term is probably degenerate at the leading order and it will be necessary to consider higher order equations to see the effects of the bending resonance of the floors. The analysis of the balance equations provided by the HPDM method is still realized in two steps: first the macroscopic description and then the inner equilibrium of the cell.

The first two significant orders of the balance of the vertical forces (SN0) and (SN $\frac{1}{2}$ ) are given below. The main difference with Section 3.1 is the multiplication of $\Lambda_{f}$ by a function $f$ depending on the frequency.

$$
\begin{aligned}
& \Lambda_{w} \tilde{\omega}_{0}^{2} \tilde{V}^{0}(x)+2 E_{w} A_{w} \tilde{V}^{0 \prime \prime}(x)=0 \\
& \Lambda_{w} \tilde{\omega}_{0}^{2} \tilde{V}^{1 / 2}(x)+2 E_{w} A_{w} \tilde{V}^{1 / 2}{ }^{\prime \prime}(x)+2 \Lambda_{w} \tilde{\omega}_{0} \tilde{\omega}_{1 / 2} \tilde{V}^{0}(x)+\Lambda_{f} f\left(\tilde{\omega}_{0}\right) \tilde{\omega}_{0}^{2} \tilde{V}^{0}(x)=0
\end{aligned}
$$

The leading order equation [Eq. (12a)] only describes the tension-compression behaviour of the walls as expected. The mass of the floors appears in the next equation [Eq. (12b)]. The other inertial terms of Eq. (12b) with $\Lambda_{w}$ come from the expansions of $V$ and $\omega^{2}$. At most of the frequencies, $f\left(\tilde{\omega}_{0}\right)=O(1)$ and Eq. (12a) is sufficient for the description of the macroscopic behaviour. However, we will see that $f\left(\tilde{\omega}_{0}\right)$ can become infinite. In that case, the in-

ertial term related to the floors is no longer negligible. Since we wish a macroscopic description which is valid for the whole frequency range of the longitudinal vibrations and takes into account the resonance of the floors, we sum Eqs. (12a) and (12b).

$$
\Lambda(\hat{\omega}) \hat{\omega}^{2} \hat{V}(x)+2 E_{w} A_{w} \hat{V}^{\prime \prime}(x)=o(\sqrt{\epsilon})
$$


with

$$
\begin{gathered}
\hat{V}(x)=\tilde{V}^{0}(x)+\tilde{V}^{1 / 2}(x) \quad ; \quad \hat{\omega}=\tilde{\omega}_{0}+\tilde{\omega}_{1 / 2} ; \Lambda(\hat{\omega})=\Lambda_{w}+\Lambda_{f} f(\hat{\omega}) \\
f(\hat{\omega})=\frac{8}{3 \pi \sqrt{\frac{\hat{\omega}}{\omega_{f 1}}}\left[\operatorname{coth}\left(\frac{3 \pi}{4} \sqrt{\frac{\hat{\omega}}{\omega_{f 1}}}\right)+\cot \left(\frac{3 \pi}{4} \sqrt{\frac{\hat{\omega}}{\omega_{f 1}}}\right)\right]}
\end{gathered}
$$

Eq. (13a) looks like Eq. (12a) but differs fundamentally by the effective mass $\Lambda(\hat{\omega})$ which depends on the frequency. The HPDM method provides the analytical expression of the function $f$. Its variations according to the frequency are plotted in Figure 3. It shows that $f(\hat{\omega}) \rightarrow 1$ when $\hat{\omega} \rightarrow 0$ as expected and that $|f(\hat{\omega})| \rightarrow+\infty$ when $\hat{\omega} \rightarrow \omega_{f(2 k-1)}$ where $\omega_{f(2 k-1)}$ are the circular frequencies of the odd normal modes in bending of the floors with two clamped ends. At most of the circular frequencies higher than $\omega_{f 1}$, we have $f(\hat{\omega})<1$, which means that the structure seems lighter because of the local resonance. Note also that $f(\hat{\omega})$ is negative just after the frequencies of the odd normal modes in bending of the floors.

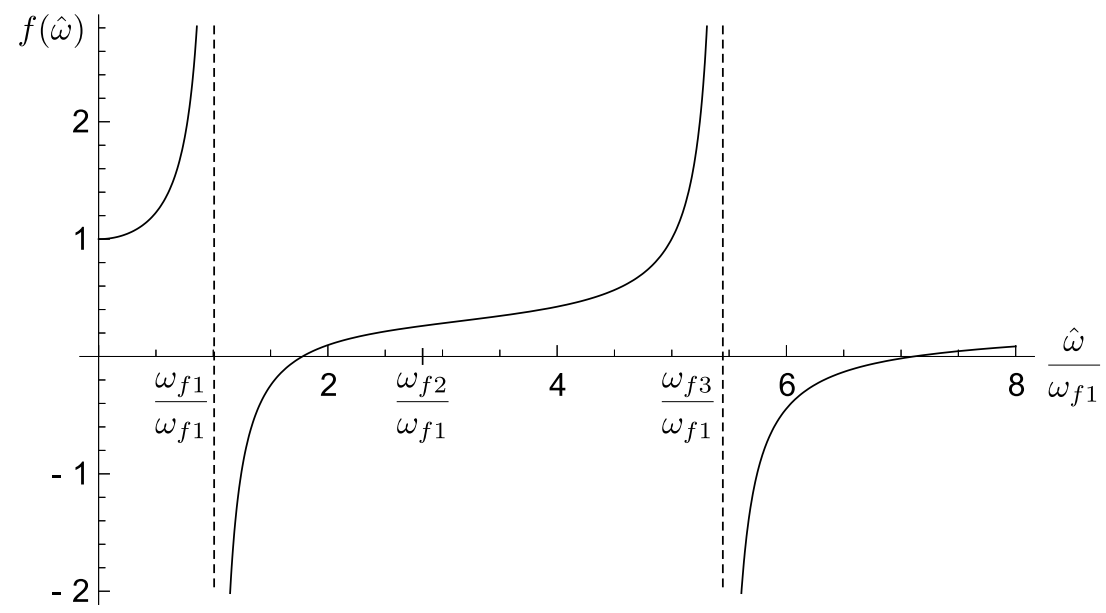

Figure 3: Variations of the function $f$ according to the dimensionless frequency $\hat{\omega} / \omega_{f 1}$. According to Eq. (37) of Appendix A, the modes of the floors correspond to the following abscissas: $\omega_{f 1} / \omega_{f 1}=3^{2} / 3^{2}=1, \omega_{f 2} / \omega_{f 1}=5^{2} / 3^{2} \approx 2.78, \omega_{f 3} / \omega_{f 1}=7^{2} / 3^{2} \approx 5.44$, etc.

As explained in [7], the effective mass differs from the real mass because the resonating floors are in relative motion compared with the walls which are in a quasi-static state. By definition, the kinematic descriptors correspond to the motion of the nodes. At low frequencies, the cell experiences a translation at the leading order and the internal relative motions are negligible. 
Consequently, the mean displacement of the nodes $\mathrm{V}$ is also the leading order displacement of every point of the cell. The sum of the inertia forces acting on the whole frame equals at the leading order the real mass of the frame multiplied by the acceleration of the nodes. When bending resonance occurs, the displacement of the nodes remains the leading order displacement of the quasi-static walls but the motion of the floors can strongly differ from the one of the nodes and some points can be in antiphase. In these conditions, the inertia forces on the walls are unchanged but the inertia forces acting on the floors vary with their deformation and then the frequency. The sum of the inertia forces acting on a floor is calculated in Appendix A [Eq. (42)]. It consists of two terms. One term depends on the node displacement $V$ and the other depends on the node rotation $\Phi$. If the term proportional to $V$ is divided by $\ell_{w}$ to have a force per unit length, we obtain $\Lambda_{f} f(\omega) \omega^{2} V$, that is to say the inertial term related to the floors in the macroscopic description [Eq. (13a)]. The term with $\Phi$ in the inertial forces on the floors probably appears in the higher order equations. Moreover, the study of the inner equilibrium of the cell [Eq. (14) afterwards] shows that $\tilde{\Phi}^{0}(x)=0$ and that the leading order is $\tilde{\Phi}^{1 / 2}(x)$.

The variations of the function $f$ plotted in Figure 3 show that the symmetry of the longitudinal kinematics excites the odd bending modes of the floors but not their even bending modes. At the frequencies of the odd bending modes, a small displacement of the nodes produces a large deformation of the floors and the inertia forces become huge. The motion of the floors is mainly in phase with the motion of the nodes at the frequencies lower than the ones of the odd modes and mainly in antiphase at the frequencies higher than the ones of the odd modes. Even if there is no resonance at the frequencies of the even bending modes, the motion of the floors is not uniform and the effective mass is smaller than the real mass.

The introduction of damping does not modify fundamentally the previous results. Figure 4 shows the variations of the real part and the imaginary part of the function $f$ when the elastic modulus is a complex number: $\hat{E}=E \mathrm{e}^{\mathrm{i} \eta}$ with $\eta=2.10^{-2}$ for the calculations. As in the undamped case, $f(\hat{\omega}) \rightarrow 1$ at low frequencies. The real part has peaks and changes its sign at the frequencies of the odd bending modes of the floors but not at the frequencies of the even bending modes. The imaginary part is very small except in the neighbourhood of the odd bending modes of the floors. At these frequencies the damping in the structure becomes very important.

After the study of the macroscopic description, we now consider the inner 


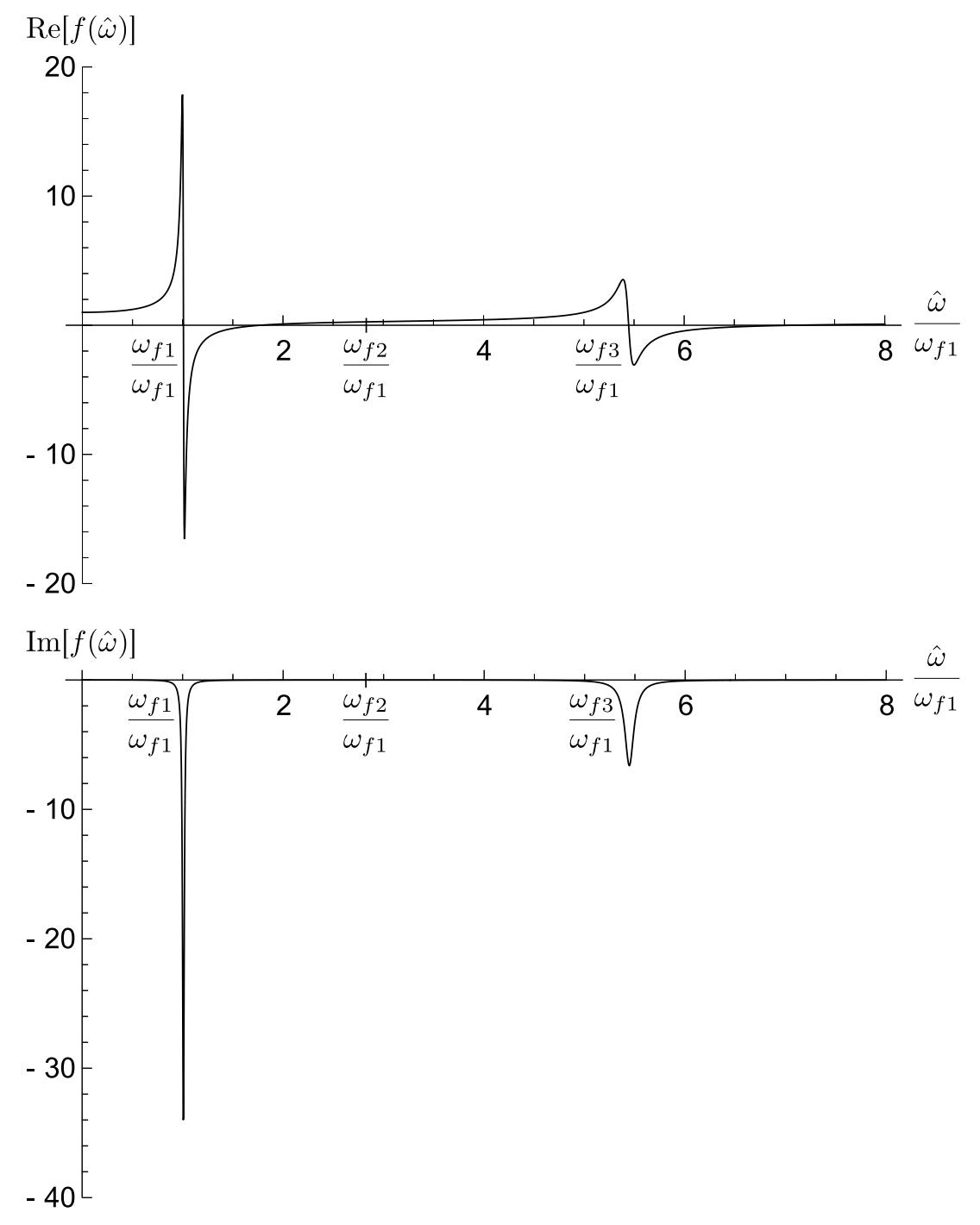

Figure 4: Variations of the real part and the imaginary part of the function $f$ according to the dimensionless frequency $\hat{\omega} / \omega_{f 1}$ in the damped case $\left(\hat{E}=E \mathrm{e}^{\mathrm{i} \eta}\right.$ with $\left.\eta=2.10^{-2}\right)$. 
equilibrium of the cell. In the quasi-static case, Eq. (11) depends on the stiffness of the elements in bending $K_{w}$ and $K_{f}$. Therefore these equations are probably modified by the local resonance. Moreover in [7], it was shown that these equations can impose additional kinematic conditions in the presence of the local resonance. The leading order of the equations (DT) and (DM) is given below:

$$
\begin{aligned}
(\mathrm{DT}) & \Rightarrow \quad \frac{12 E_{f} A_{f}}{\ell_{w} \ell_{f}} \tilde{\Delta}^{3}(x)+3 K_{w} \tilde{\Phi}^{1 / 2 \prime}(x)=0 \\
(\mathrm{DM}) & \Rightarrow \quad 3 K_{w} \tilde{\Phi}^{1 / 2}(x)-\Lambda_{f} \ell_{f} g\left(\tilde{\omega}_{0}\right) \tilde{\omega}_{0}^{2} \tilde{V}^{0}(x)=0 \\
\text { with } g(\omega) & =\frac{48}{(3 \pi)^{2} \frac{\omega}{\omega_{f 1}}} \frac{\left[\operatorname{coth}\left(\frac{3 \pi}{4} \sqrt{\frac{\omega}{\omega_{f 1}}}\right)-\cot \left(\frac{3 \pi}{4} \sqrt{\frac{\omega}{\omega_{f 1}}}\right)\right]}{\left[\operatorname{coth}\left(\frac{3 \pi}{4} \sqrt{\frac{\omega}{\omega_{f 1}}}\right)+\cot \left(\frac{3 \pi}{4} \sqrt{\frac{\omega}{\omega_{f 1}}}\right)\right]} \\
& =\frac{6 f(\omega)}{3 \pi \sqrt{\frac{\omega}{\omega_{f 1}}}}\left[\operatorname{coth}\left(\frac{3 \pi}{4} \sqrt{\frac{\omega}{\omega_{f 1}}}\right)-\cot \left(\frac{3 \pi}{4} \sqrt{\frac{\omega}{\omega_{f 1}}}\right)\right]
\end{aligned}
$$

The equation (DT) involves the axial force in the floors and the shear force in the walls. This equation is not affected by the local resonance because these forces are expanded normally. (This is also true if we consider the higher orders.) The only difference with Eq. (11) of Section 3.1 is the modification of the orders of magnitude due to the introduction of a thickness contrast between the elements.

On the contrary, the equation (DM) in the quasi-static case contains an inertial term from the floors and the stiffness in bending of the elements. In the presence of the local resonance of the floors, the inertial term depends on the frequency as indicated in Eq. (14). This term is different from the effective mass in the macroscopic description [Eq. (13)] but it is proportional to the function $f$ and we also have $g(\omega) \rightarrow 1$ at low frequencies. As a result, the inertial term of Eq. (14) becomes infinite at the frequencies of the odd bending modes of the floors and it changes its sign. The comparison of the expression of the function $g$ with Eq. (36) of Appendix A shows that this function vanishes at the frequencies of the even bending modes. The stiffness in bending of the floors $K_{f}$ does not appear in Eq. (14) because it is now negligible compared to $K_{w}$. The study of the higher orders shows that 
this term also depends on the frequency. It becomes infinite at the same frequencies as the functions $g$ and $f$, that is to say the frequencies of the odd bending modes of the floors. For this structure, the inner equilibrium of the cell does not introduce new singularities.

\section{Some consequences of the local resonance}

This section illustrates the consequences of the local resonance and the variations of the effective mass by considering two problems: the free and forced vibrations of a structure governed by the following equations:

$$
\begin{gathered}
\Lambda(\omega) \omega^{2} V(x)+2 E_{w} A_{w} V^{\prime \prime}(x)=0 \quad \text { with } \Lambda(\omega)=\Lambda_{w}+\Lambda_{f} f(\omega) \\
\text { and } f(\omega)=\frac{8}{3 \pi \sqrt{\frac{\omega}{\omega_{f 1}}}\left[\operatorname{coth}\left(\frac{3 \pi}{4} \sqrt{\frac{\omega}{\omega_{f 1}}}\right)+\cot \left(\frac{3 \pi}{4} \sqrt{\frac{\omega}{\omega_{f 1}}}\right)\right]}
\end{gathered}
$$

For the forced vibration, damping is introduced. The structure is clamped at the bottom and free at the top. Its total height is $H=N \ell_{w}$. Then, the atypical behaviours are confirmed by finite element simulations.

\subsection{Free vibration}

We first study the normal modes of the structure. In this case, the solution of Eq. (15) is:

$$
\hat{V}(x)=B \sin (\alpha x) \quad \text { with } \quad \alpha^{2}=\frac{\Lambda(\hat{\omega}) \hat{\omega}^{2}}{2 E_{w} A_{w}} \quad \text { and } \quad \cos (\alpha H)=0
$$

Thus the natural frequencies are given by Eq. (17).

$$
\alpha_{k} H=\frac{(2 k-1) \pi}{2} \Rightarrow \frac{\Lambda(\hat{\omega}) \hat{\omega}^{2}}{2 E_{w} A_{w}}\left(\frac{2 H}{\pi}\right)^{2}=(2 k-1)^{2}
$$

In the absence of the local resonance, the effective mass $\Lambda(\omega)$ is constant and there is only one solution $\omega_{k}$ for each value of $k$.

From the expression of the effective mass given in Eq. (15) and the variations of the function $f$ plotted in Figure 3, we deduce that the local resonance has three effects on the modal properties of a reticulated structure. The greater the proportion of the mass in the floors is, the more important the effects of the resonance are. 
1. Modification of the resonance frequencies: Using the effective mass instead of the real mass gives different values for the natural frequencies of the structure.

2. No uniqueness of the modal frequencies: Since the function $f$ does not monotonically increase with the frequency, there are several solutions $\omega_{k}$ for each value of $k$. This means that the structure has the same macroscopic mode shape at several frequencies. However, at the local scale, the deformation of the floors is different. They move in-phase with the walls below $f_{f 1}$, the frequency of the first bending mode of the floors, and in antiphase with the walls above $f_{f 1}$. At higher frequencies, the floors have a more complex deformation.

3. High density of modes: Because of the great variations of the function $f$ in the neighbourhood of the frequencies of the odd bending modes of the floors, Eq. (17) has a solution close to these frequencies for each value of $k$. Consequently there is a large number of modes in a narrow frequency range. At the frequencies slightly higher than the frequencies of the odd modes of the floors, the effective mass is negative and there is a frequency bandgap without any longitudinal mode.

Usually the condition of scale separation limits homogenization to low frequencies. Since several modes of the structure can have the same wavelength, some high frequency modes are correctly described by homogenization and some low frequency modes does not respect the condition of scale separation. These modes have a frequency close to the frequencies of the odd bending modes of the floors. Because of the dispersion introduced by the local resonance, the scale ratio $\epsilon$ does not monotonically increase with the frequency. There is an alternation of frequencies at which homogenization applies and frequencies at which there is no scale separation.

\subsection{Forced vibration}

A harmonic vertical motion of amplitude $V_{0}$ is now imposed at the bottom of the structure. We are interested in the evolution with the frequency of the amplitude of $V(H)$ the displacement at the top. The solution of Eq. (15) depends on the sign of the effective mass as indicated in Eq. (18).

$$
\begin{array}{ll}
\Lambda(\omega) \geq 0 \quad & \Rightarrow \quad V(x)=V_{0}[\cos (\alpha x)+\tan (\alpha H) \sin (\alpha x)] \\
& \Rightarrow \quad H(\omega)=\frac{V(H)}{V_{0}}=\frac{1}{\cos (\alpha H)}
\end{array}
$$




$$
\begin{aligned}
\Lambda(\omega)<0 \quad \Rightarrow \quad V(x) & =V_{0}[\cosh (\alpha x)-\tanh (\alpha H) \sinh (\alpha x)] \\
\Rightarrow \quad H(\omega) & =\frac{V(H)}{V_{0}}=\frac{1}{\cosh (\alpha H)} \\
\text { with } & \alpha^{2}=\frac{|\Lambda(\omega)| \omega^{2}}{2 E_{w} A_{w}}
\end{aligned}
$$

When the effective mass is positive, the transfer function $|H(\omega)|$ is always greater than 1 and the imposed motion is amplified by the structure. Moreover, $|H(\omega)| \rightarrow+\infty$ as $\omega$ approaches the natural frequencies of the structure determined in Section 4.1. When the effective mass is negative, the transfer function $|H(\omega)|$ is always less than 1 and the imposed motion is attenuated by the structure. This corresponds to a bandgap. The attenuation is more important if $|\Lambda(\omega)|$ is great, that is to say at the beginning of the bandgaps for the frequencies slightly higher than the frequencies of the odd modes of the floors. When the effective mass vanishes, the transfer function is equal to 1 and the displacement at the top is identical to the displacement at the bottom.

\subsection{Example}

The results presented in Sections 4.1 and 4.2 are illustrated by the study of a particular structure. It is an academic example which corresponds to the normalization of Eq. (8). The application of the results to more realistic structures is discussed in Section 5 which also contains some guidelines for the design of new structures with prescribed properties. The chosen example is a frame structure as in Figure 1 with $N=15$ levels. The walls and the floors have the same length $\ell_{w}=\ell_{f}=3 \mathrm{~m}$ and the total height of the structure is $H=N \ell_{w}=45 \mathrm{~m}$. The thickness to length ratios correspond to the orders of magnitude of Eq. (8). In [10,11], it was shown that the scale ratio can be estimated by $\epsilon \approx \pi /(2 N)$ for the first macroscopic mode of a structure. The walls are made of concrete but the density of the floors is increased in order to have $\Lambda_{f} \approx \Lambda_{w}$ and to increase the influence of their resonance. The 
characteristics of the structure are summarized in Eq. (19).

$$
\begin{array}{rlrl}
N & =15 & H & =45 \mathrm{~m} \\
\ell_{w} & =\ell_{f}=3 \mathrm{~m} & h_{w} & =h_{f}=1 \mathrm{~m} \\
a_{w} & =0.971 \mathrm{~m} & a_{f} & =0.314 \mathrm{~m} \\
E_{w} & =E_{f}=30000 \mathrm{MPa} & \nu_{w} & =\nu_{f}=0.2 \\
\rho_{w} & =2300 \mathrm{~kg} \cdot \mathrm{m}^{-3} & \rho_{f} & =14225 \mathrm{~kg} \cdot \mathrm{m}^{-3}
\end{array}
$$

The frequencies of the bending modes of the floors with two clamped ends are determined thanks to Eq. (35) of Appendix A. The frequencies of the first two odd modes are $f_{f 1}=52.08 \mathrm{~Hz}$ and $f_{f 3}=281.44 \mathrm{~Hz}$. The frequency of the first even mode is $f_{f 2}=143.56 \mathrm{~Hz}$. The real mass of the structure is $\Lambda=8933.25 \mathrm{~kg} \mathrm{~m}^{-1}$. The variations of the effective mass in the neighbourhood of the first resonance of the floors are plotted in Figure 5. Note that the difference between the real mass and the effective mass becomes significant at approximatively $25 \mathrm{~Hz}$ which is much less than $f_{f 1}$. The bandgap corresponds to the frequencies with a negative effective mass. For this structure, it is between $f_{f 1}=52.08 \mathrm{~Hz}$ and $64.29 \mathrm{~Hz}$.

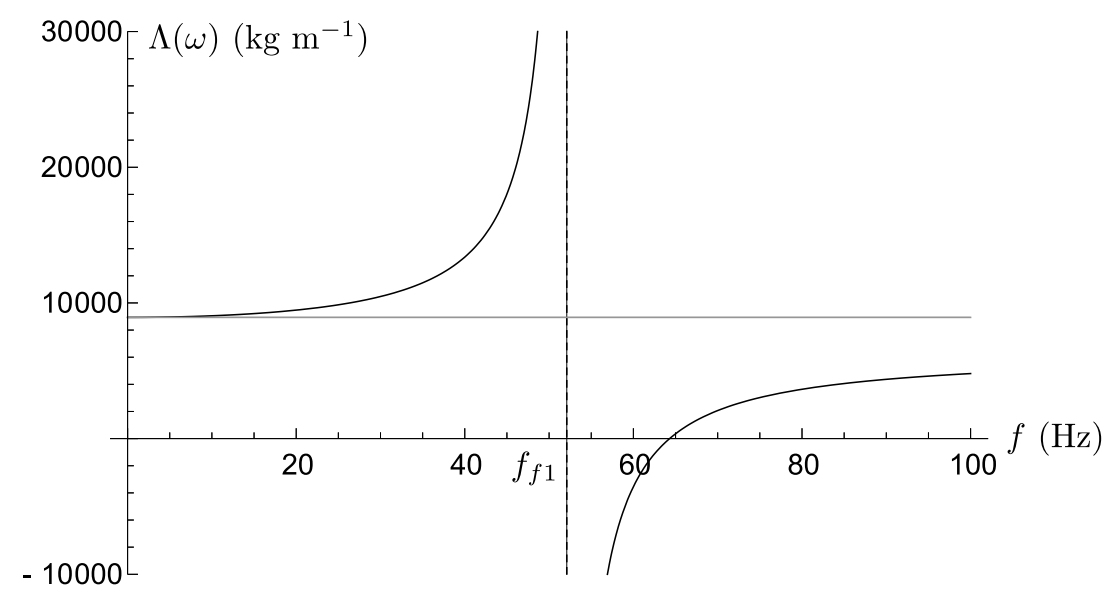

Figure 5: Variations of the effective mass according to the frequency in the neighbourhood of the first resonance of the floors. The grey horizontal line indicates the value of the real mass.

We first calculate the natural frequencies of the structure. Eq. (17) is solved graphically in Figure 6. If the effects of the local resonance are ignored by using the real mass instead of the effective mass, we obtain the frequencies 


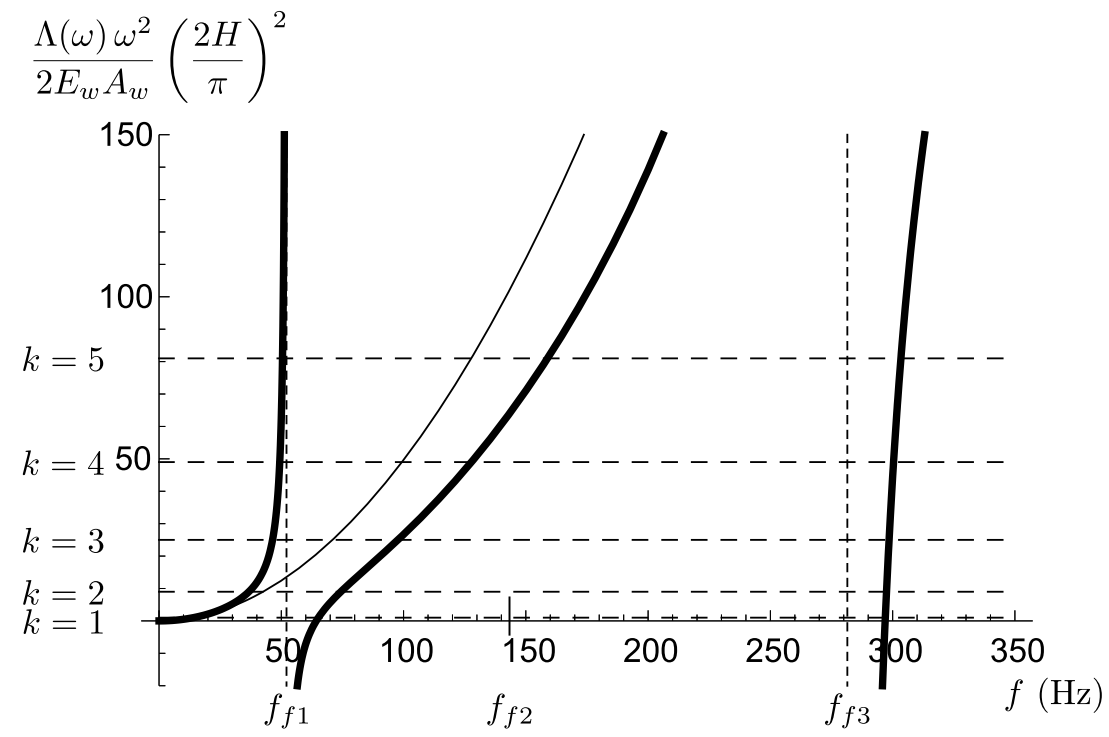

Figure 6: The thin line is obtained by replacing the effective mass by the real mass. The thick line takes into account the resonance of the floors. The horizontal dashed lines indicate the first values of $(2 k-1)^{2}$. The natural frequencies of the structure are the abscissas of the intersections of the continuous curves with the dashed lines.

given in the second column of Table 1. As these frequencies are in the same range as the frequencies of the bending modes of the floors, the variations of the effective mass cannot be neglected. Including the effects of the local resonance leads to the frequencies given in the third column of Table 1. As expected, there are now several frequencies for each value of $k$ and they are different from the frequencies of the second column of Table 1.

These results are confirmed by simulations made with the finite element code CESAR-LCPC. The longitudinal modes are determined for two structures. For the first one, the elements behave as Euler-Bernoulli beams as in the HPDM method. For the second one, the elements behave as Timoshenko beams which is more realistic for the chosen thickness. The natural frequencies of both structures are also given in Table 1. There is an excellent agreement with the homogenized model. The use of Timoshenko beams slightly modifies the frequencies but the mode shapes are identical. The mode shapes are presented in Figure 7 and they are compared with the homogenized model [Eq. (16)] in Figure 8.

The effects of the local resonance are also visible on the transfer function. Using the real mass instead of the effective mass leads to an erroneous 


\begin{tabular}{|c|c|c|c|c|}
\hline $\mathrm{k}$ & $\begin{array}{c}\text { Resonance of the } \\
\text { floors neglected } \\
{[\text { Eq. }(10)]}\end{array}$ & $\begin{array}{c}\text { Resonance of the floors } \\
\text { taken into account } \\
{[\text { Eq. }(15)]}\end{array}$ & $\begin{array}{c}\text { CESAR-LCPC with } \\
\text { Euler-Bernoulli } \\
\text { beams }\end{array}$ & $\begin{array}{c}\text { CESAR-LCPC with } \\
\text { Timoshenko } \\
\text { beams }\end{array}$ \\
\hline 1 & 14.19 & $14.00-65.14-297.0$ & $13.76-64.83$ & $13.73-61.35$ \\
\hline 2 & 42.56 & $36.73-74.35-297.5$ & $35.99-74.22$ & $35.12-71.71$ \\
\hline 3 & 70.94 & $46.42-97.68-298.5$ & $45.46-97.37$ & $43.32-96.04$ \\
\hline 4 & 99.31 & $49.36-127.8-300.4$ & $48.28-127.0$ & $45.66-125.5$ \\
\hline 5 & 127.69 & $50.49-158.9-303.4$ & $49.76-157.5$ & $46.93-154.2$ \\
\hline
\end{tabular}

Table 1: Comparison of the natural frequencies $(\mathrm{Hz})$ of the structure estimated thanks to the homogenized models and with the finite element code CESAR-LCPC.

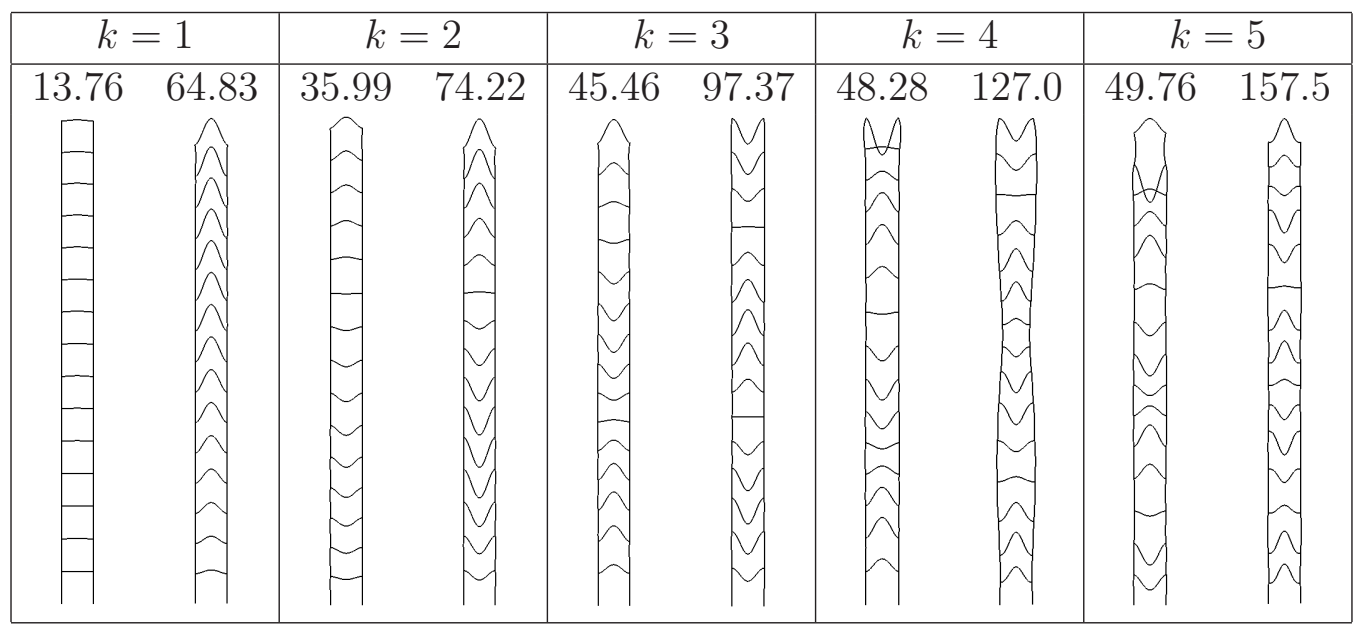

Figure 7: Mode shapes and natural frequencies $(\mathrm{Hz})$ calculated with the finite element code CESAR-LCPC. For each value of $k$, the walls and the floors move in-phase at the lower frequency and in antiphase at the higher frequency. 

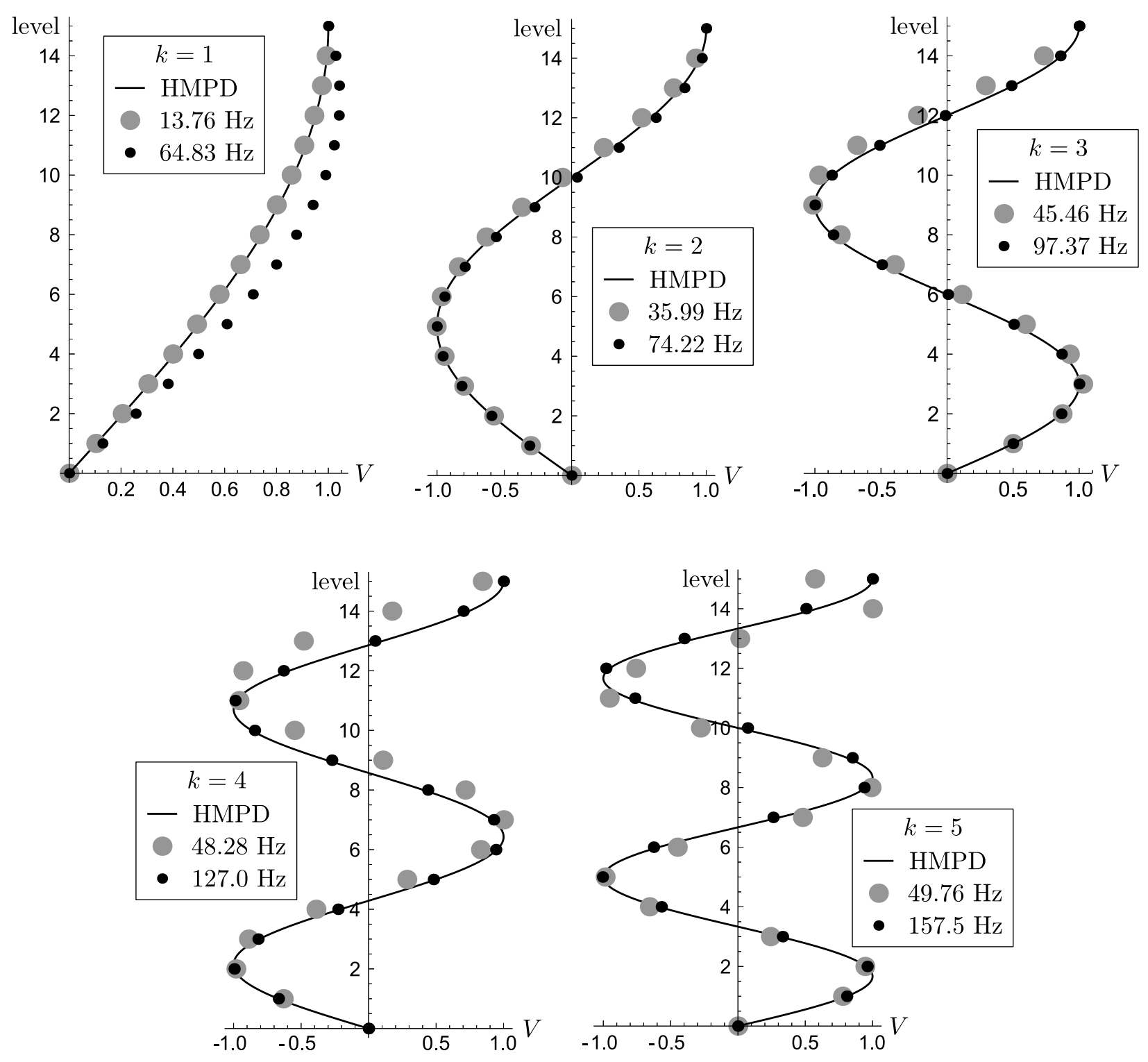

Figure 8: Comparison of the mean vertical displacement of the nodes estimated with the homogenized model [Eq. (16)] and the finite element code CESAR-LCPC. 


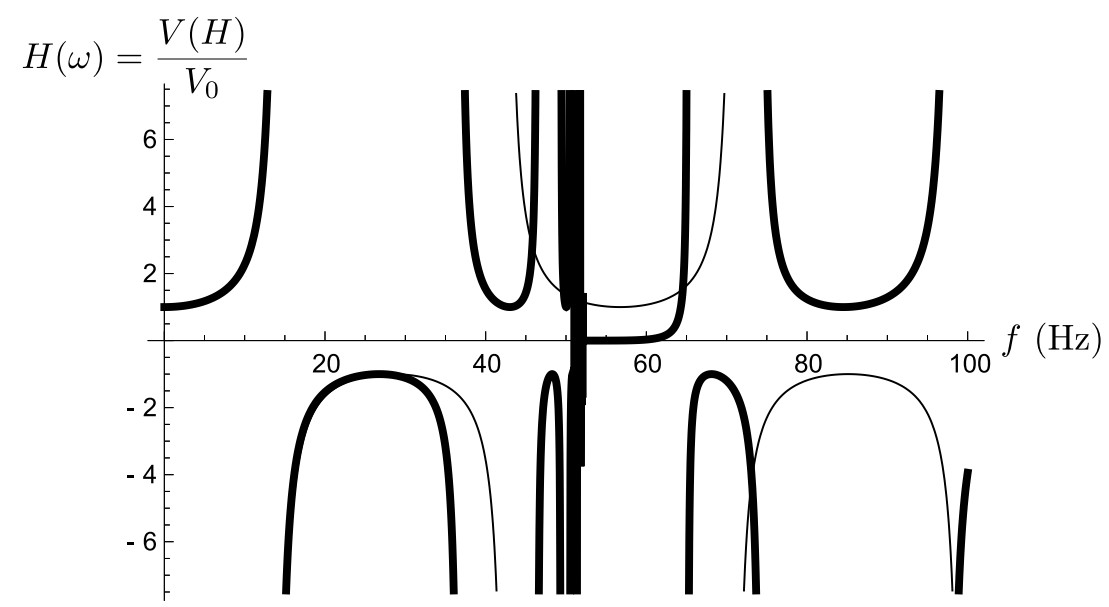

Figure 9: Comparison of the transfer function calculated with the real mass (thin line) and the transfer function calculated with the effective mass (thick line)

description above $25 \mathrm{~Hz}$ (Figure 9). As expected, the two transfer functions have peaks at different frequencies and the local resonance causes a bandgap between $f_{f 1}=52.08 \mathrm{~Hz}$ and $64 \mathrm{~Hz}$.

The transfer function computed with the finite element method is almost identical to the transfer function given by the homogenized model with local resonance (Figure 10). The main difference is the number of peaks just before the resonance of the floors. In this frequency range the peaks are concentrated because of the great variations of the effective mass. However, a lot of the peaks of the homogenized transfer function do not correspond to real modes of the structure. Indeed, the homogenization process replaces the structure by an equivalent beam which has an infinite number of degrees of freedom and therefore an infinite number of longitudinal modes. On the contrary, the studied structure has only 15 possible macroscopic mode shapes. As a result, the real transfer function has less peaks and the bandgap begins at slightly lower frequencies. An example of the deformation of the structure inside the bandgap is also presented in Figure 10. The first floors experience large deformations because of the resonance but the energy is not transferred to the upper levels. From the fourth floor, there is no more vibration.

The introduction of damping does not modify fundamentally the results. As in Section 3.2 a complex elastic modulus $E_{f}=E_{w}=E \mathrm{e}^{\mathrm{i} \eta}$ is used in the homogenized model [Eq. (18)]. The modulus of the transfer function is plotted in Figure 11 for $\eta=2.10^{-2}$. The main differences with Figure 10 are that 
(a)

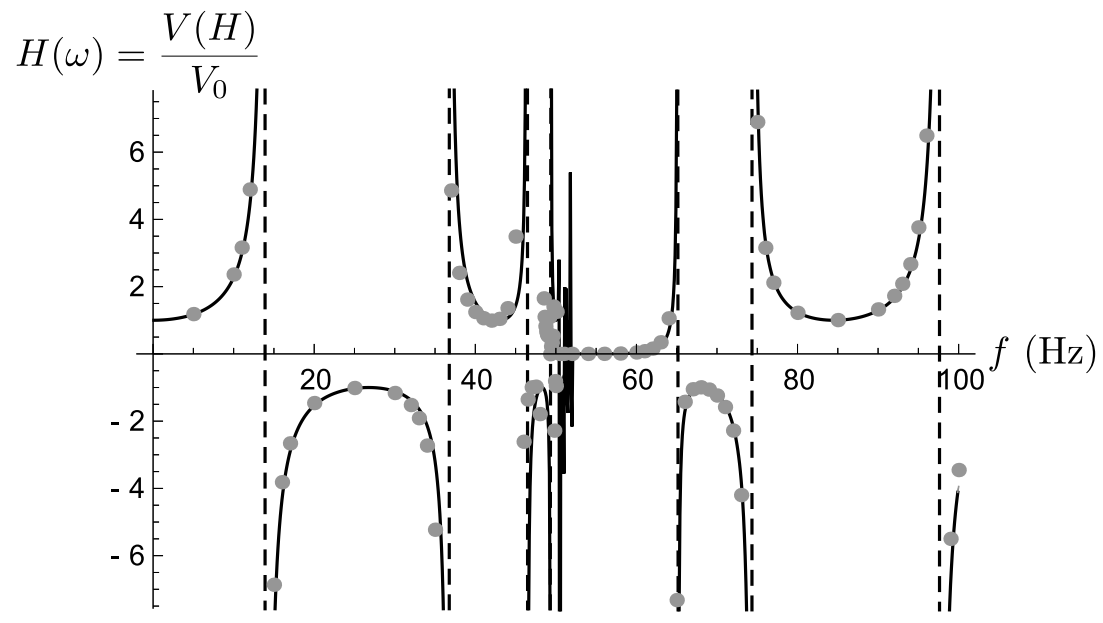

(b)



Figure 10: (a) Transfer function of the studied structure. The continuous curve corresponds to the homogenized model [Eq. (18)] and the points to the finite element simulations; (b) Deformation of the structure at $52 \mathrm{~Hz}$.

the frequency bandgap is larger and the peaks of the transfer function before the resonance no longer exist. Finite element simulations are still in good agreement with the transfer function of the homogenized model (Figure 11).

Even if the homogenized model is limited to the leading order, the comparison with the finite element method shows that it gives very good results in the whole considered frequency range for a much smaller cost. There is only a small discrepancy at the resonance frequency of the floors. A possible explanation is that this frequency range corresponds to the higher modes of the structure and that the scale ratio $\epsilon$ is less small. Moreover, the higher order equations probably contain correctors which depend on the frequency and become more important in this frequency range.

\section{Potential applications}

\subsection{Building dynamics}

As building dynamics was the initial motivation of this work, it is the first application we consider. The vertical modes of buildings and the bending modes of their floors can be excited by ground vibration due to construction work or road and railway traffic $[13,14]$ and can cause annoyance to the occupants. Moreover, during an earthquake, the vertical motion can be higher than the horizontal motion in the vicinity of the fault rupture. This 

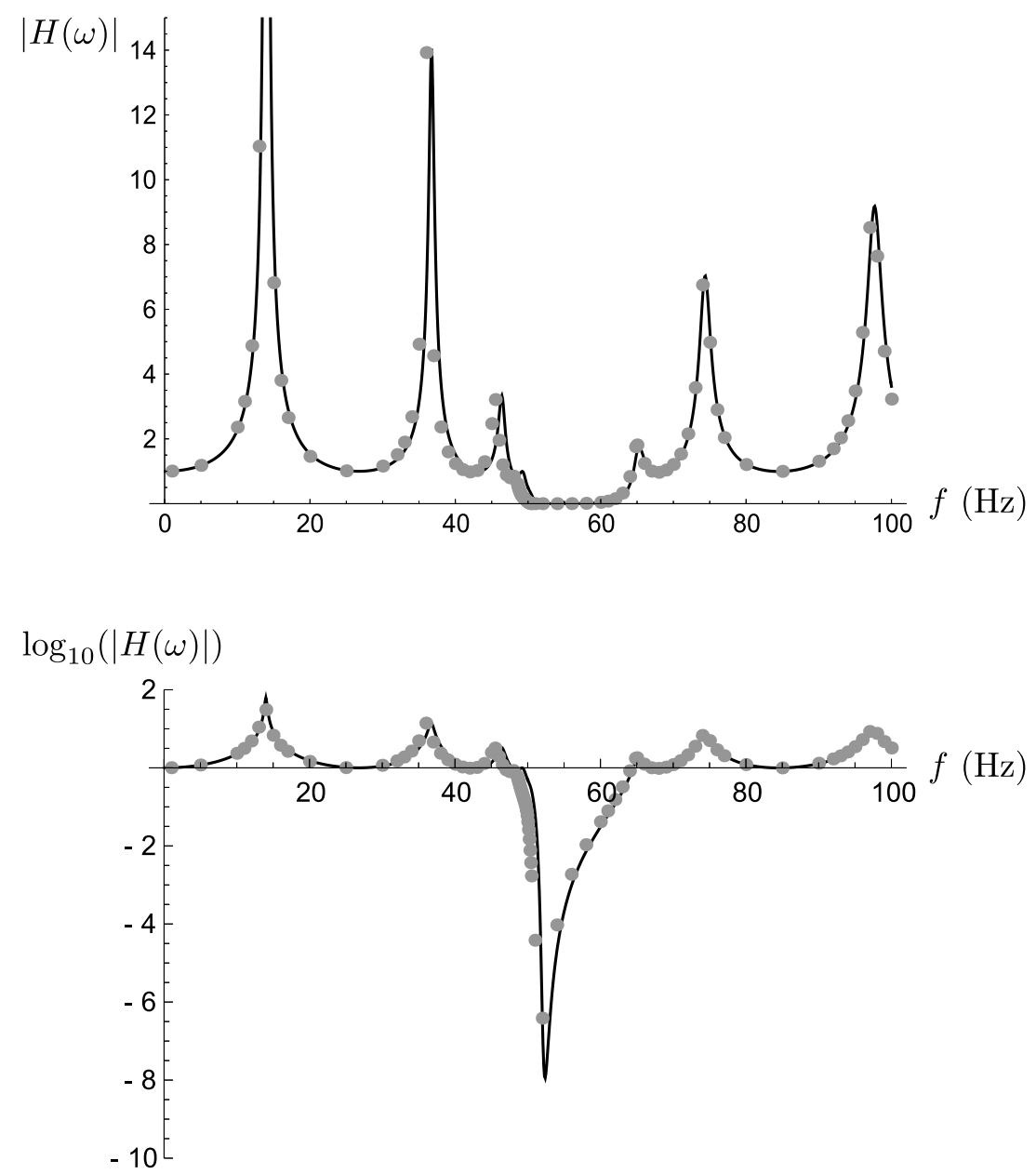

Figure 11: Modulus of the transfer function of the studied structure with damping $\left(E_{f}=E_{w}=E \mathrm{e}^{\mathrm{i} \eta}\right.$ with $\left.\eta=2 \cdot 10^{-2}\right)$. The continuous curves correspond to the homogenized model [Eq. (18)] and the points to the finite element simulations. 
can lead to the failure of the vertical bearing elements or of the floors [15]. The example of Section 4.3 is a good illustration of the phenomenon studied in the paper but it differs from real buildings in the following points which will be examined in this section:

- The thickness of the elements and the density of the floors are not realistic.

- Buildings have a more complex geometry and each level contains several walls and floors.

- Buildings are not perfectly clamped at the bottom but are supported by a viscoelastic soil.

To show that the bending resonance of the floors can also have an effect on the vertical modes of some buildings, we present a second example with more realistic dimensions. We keep the simple geometry of Figure 1 and the same boundary conditions. The structure is clamped at the bottom and free at the top. All the elements are made of concrete. The new characteristics are summarized in Eq. (20).

$$
\begin{aligned}
N & =20 & & H=N \ell_{w}=50 \mathrm{~m} \\
\ell_{w} & =2.50 \mathrm{~m} & & \ell_{f}=6 \mathrm{~m} \\
a_{w} & =0.30 \mathrm{~m} & & a_{f}=0.20 \mathrm{~m} \\
h_{w} & =h_{f}=1 \mathrm{~m} & & \rho_{w}=\rho_{f}=2300 \mathrm{~kg} \cdot \mathrm{m}^{-3} \\
E_{w} & =E_{f}=30000 \mathrm{MPa} & & \nu_{w}=\nu_{f}=0.2 \\
\Rightarrow \quad \Lambda_{w} & =1380 \mathrm{~kg} \cdot \mathrm{m}^{-1} & & \Lambda_{f}=1104 \mathrm{~kg} \cdot \mathrm{m}^{-1}
\end{aligned}
$$

In this case, the frequencies of the bending modes of the floors with two clamped ends [Eq. (35)] are $f_{f 1}=20.62 \mathrm{~Hz}, f_{f 2}=56.85 \mathrm{~Hz}$ and $f_{f 3}=111.45 \mathrm{~Hz}$. The natural frequencies of the structure calculated by using the real mass instead of the effective mass are given in the second column of Table 2. As these frequencies are in the same range as the frequencies of the bending modes of the floors, the effect of their resonance cannot be ignored. This leads to the frequencies given in the third column of Table 2 which are confirmed by finite element simulations. The transfer function is also affected by the bending resonance of the floors. However the mass of the floors is now $20 \%$ smaller than the mass of the walls [Eq. (20)] and the bandgaps will probably be narrower. The comparison of the transfer functions computed with the 


\begin{tabular}{|c|c|c|r|}
\hline $\mathrm{k}$ & $\begin{array}{c}\text { Resonance of the } \\
\text { floors neglected } \\
{[\text { Eq. }(10)]}\end{array}$ & $\begin{array}{c}\text { Resonance of the floors } \\
\text { taken into account } \\
{[\text { Eq. }(15)]}\end{array}$ & CESAR-LCPC \\
\hline 1 & 13.46 & $12.42-26.81-116.6$ & $12.16-26.20-112.1$ \\
\hline 2 & 40.38 & $19.70-50.21-117.7$ & $-50.03-113.0$ \\
\hline 3 & 67.30 & $20.31-78.68-121.2$ & $-77.88-116.3$ \\
\hline 4 & 94.22 & $20.47-99.06-133.4$ & $-96.28-132.0$ \\
\hline 5 & 121.1 & $20.53-106.4-158.4$ & $-101.6-157.9$ \\
\hline
\end{tabular}

Table 2: Comparison of the natural frequencies $(\mathrm{Hz})$ of the structure estimated thanks to the homogenized models and with the finite element code CESAR-LCPC.

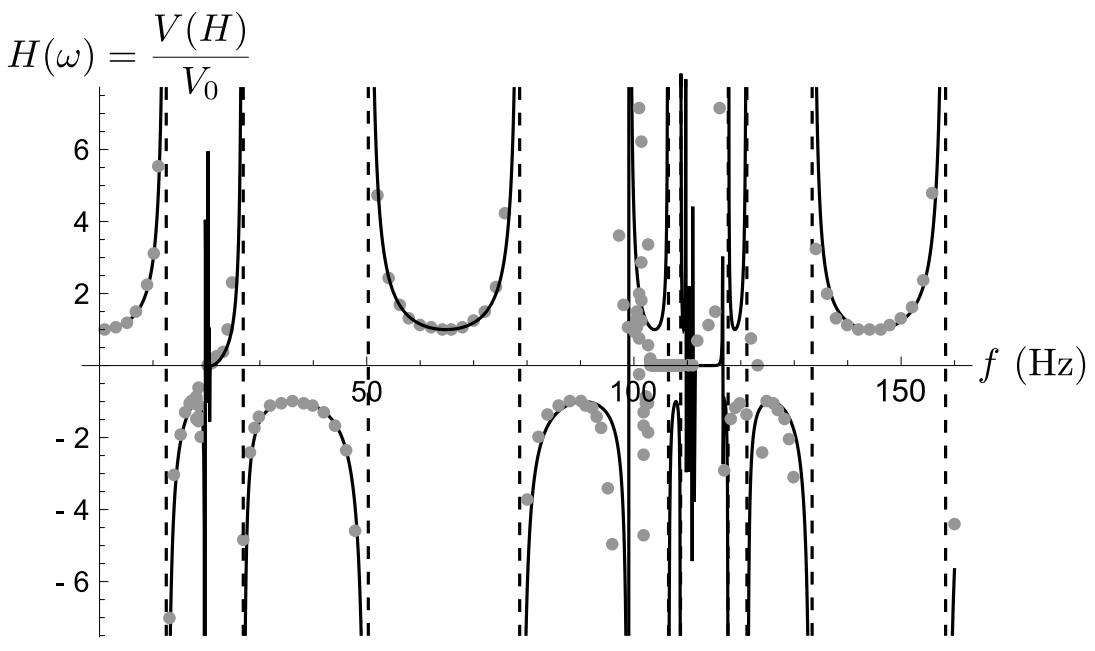

Figure 12: Transfer function of the studied structure. The continuous curve corresponds to the homogenized model [Eq. (18)] and the points to the finite element simulations. 


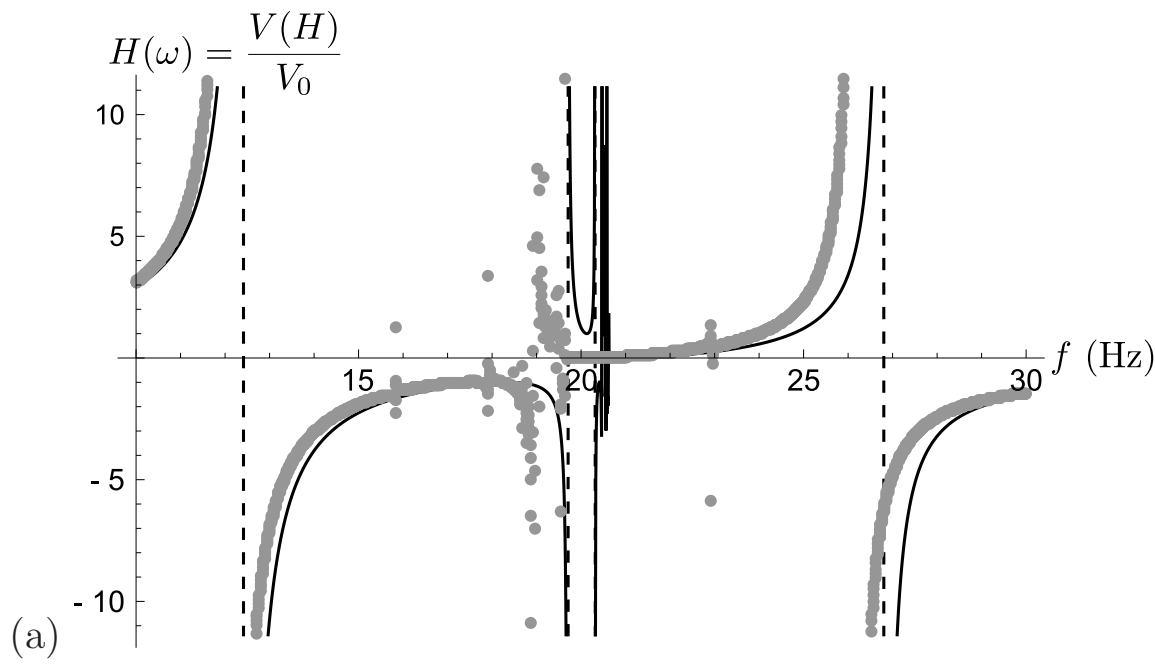

(b)
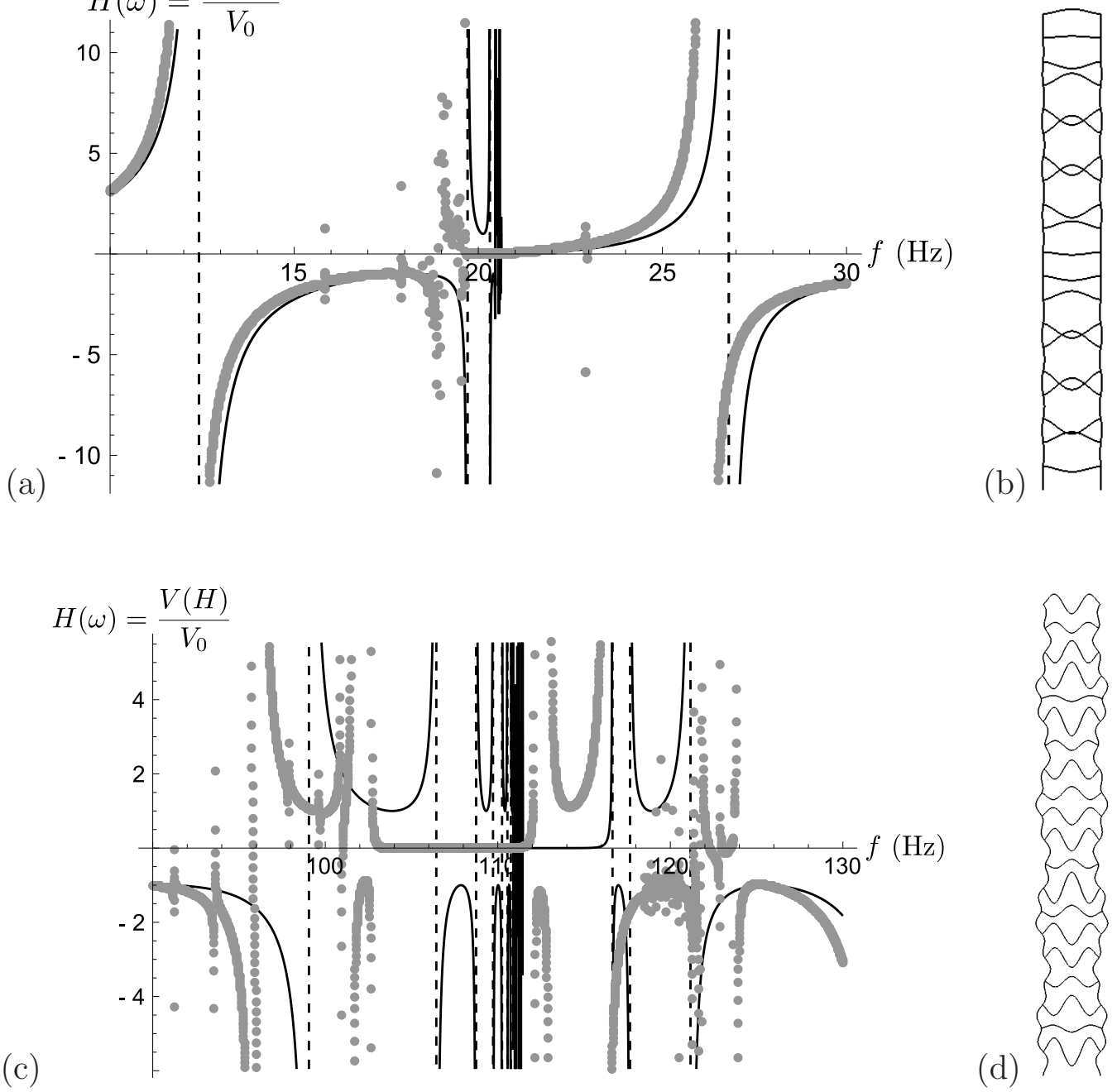

Figure 13: (a) and (c) Transfer function of the studied structure close to the natural frequencies of the floors. The continuous curve corresponds to the homogenized model [Eq. (18)] and the points to the finite element simulations; (b) and (d) Mode shapes at $18.56 \mathrm{~Hz}$ and $99.61 \mathrm{~Hz}$ respectively. 
homogenized model and the finite element method is presented in Figures 12 and 13 .

All these results show that the global behaviour of the structure is well reproduced by the homogenized model even if the dimensions of the elements do not correspond exactly to the normalization. The model is slightly less accurate close to the frequency of the third bending mode of the floors because the higher order terms are neglected. However the difference in frequency between the two methods is always less than $5 \%$. Another difference visible in Figure 13 is the existence of very localized peaks on the transfer function which are not predicted by the homogenized model. These peaks are associated to modulated modes as the two examples of Figure 13. Each family of modulated modes appears in a narrow frequency range close to a natural frequency of the basic frame. The frames are deformed according to the associated mode shape and the amplitude of their deformation varies along the structure. As the kinematics of such modes is not taken into account by the HPDM method, the homogenized model cannot predict their existence. However other homogenization methods have been developed to describe the modulated modes $[16,17,18,19,20,21]$. During the calculation of the modes with the finite element method, the large number of modulated modes between 19 and $20 \mathrm{~Hz}$ made the identification of the higher vertical modes difficult. This is the reason why the last column of Table 2 is not complete.

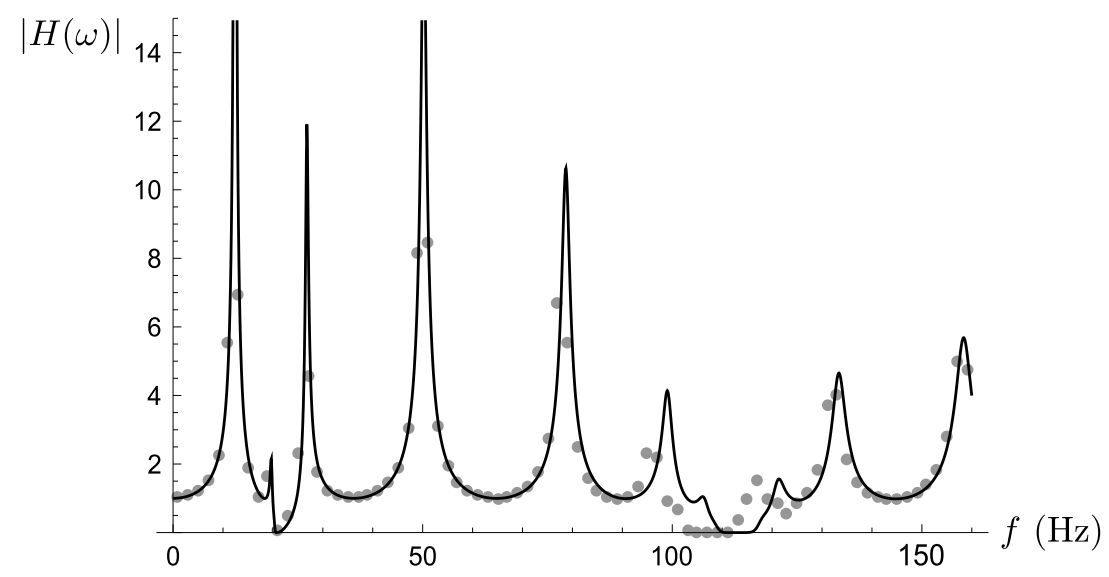

Figure 14: Modulus of the transfer function of the studied structure with damping $\left(E_{f}=E_{w}=E \mathrm{e}^{\mathrm{i} \eta}\right.$ with $\left.\eta=2 \cdot 10^{-2}\right)$. The continuous curve corresponds to the homogenized model [Eq. (18)] and the points to the finite element simulations. 

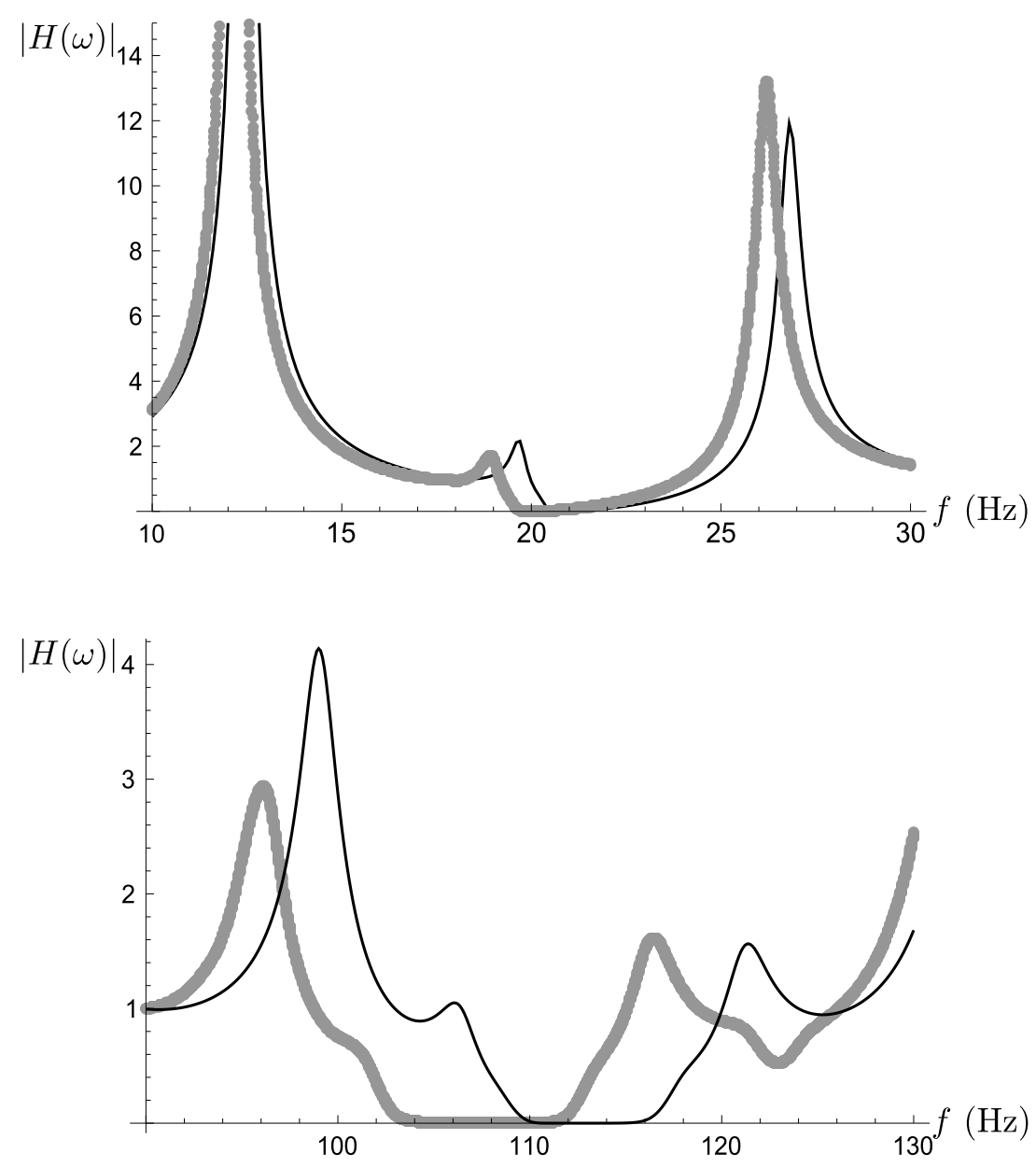

Figure 15: Modulus of the transfer function of the studied structure with damping close to the natural frequencies of the floors. The continuous curves correspond to the homogenized model [Eq. (18)] and the grey points to the finite element simulations. 
The modulus of the transfer function of a damped structure is plotted in Figure 14 and 15. As in Section 4.3, a complex elastic modulus is used: $E_{f}=E_{w}=E \mathrm{e}^{\mathrm{i} \eta}$ with $\eta=2.10^{-2}$. In this case, the modulated modes no longer create peaks on the transfer function and there is an excellent agreement between the homogenized model and the finite element simulations.

In this example, the walls and the floors have different lengths for two reasons. The first one is that the mass of the floors must be sufficiently important to see the effect of their resonance. If the contribution of the resonating elements to the total mass of the structure is negligible, the peaks of the effective mass are very narrow and the bending resonance has almost no influence. In particular, the natural frequencies of the structure are hardly modified. The second reason is that the model proposed in this paper is valid only if the bending stiffness of the walls is much greater than the bending stiffness of the floors. If this condition is not respected, we must consider the resonance of the whole frame and the behaviour is more complex [12]. This limitation comes from the simple geometry of the studied structures. As only one floor is connected to each node, the walls must be very stiff to balance the moment in the floor which becomes very important at its resonance. For a structure with several identical floors as in Figure 16(a), two floors are connected to the inner nodes and the moments are balanced. In this case, the proposed model probably remains valid even if the stiffness in bending of the walls is smaller. However, if the floors connected to a node have different properties, their natural frequencies are different and the balance of the moments depends on the bending stiffness of the walls.

Finally, the underlying soil plays an important role in the response of a real building by modifying the boundary conditions. Nevertheless, the soil-structure interaction does not change the properties of the structure described in this paper. Removing the clamped condition at the bottom can decrease the natural frequencies of the structure but not the ones of the floors. To evaluate the influence of the bending resonance on the modal properties of a real building, it is therefore necessary to compare the frequencies of the vertical modes of the soil-structure system with the frequencies of the bending modes of the floors. Examples of simulations which include the soilstructure interaction and the resonance of the floors can be found in [14]. As for the transfer function of the structure, it does not depend on the boundary condition at the bottom. 


\subsection{Design of new structures}

The results presented in this paper are not limited to building dynamics. They apply to various reticulated systems whatever their material and dimension. The great advantage of the HPDM method is that it is completely analytic. Then, even if the method is implemented on only two class of structures (defined in Eqs. (7) and (8) by the associated normalization), we know the expression of the effective mass and we can compare the modal frequencies of the whole structure and the ones of the "floors". Therefore the role of each parameter can be easily identified. The design of a structure with local resonance is based on the two following points:

- The bending wavelength in the frequency range of interest must have the same order as the length of the elements. With the notation of Appendix A, this condition is:

$$
\lambda_{b}=O(\ell) \quad \text { or } \quad \beta \ell=O(1)
$$

Moreover the frequencies of the odd bending modes of the "floors" are the lower limits of the bandgaps.

- The width of the frequency ranges with visible effects of the local resonance (including the bandgaps) depends on the proportion of the mass in the resonating elements. The greater the resonating mass is, the wider the frequency range is.

To achieve this for the example of Section 4.3, we chose to use the thickness to length ratio of the elements. We imposed that their orders of magnitude compared with the scale ratio $\epsilon$, which only depends on the number of cells, were the same as in the study with the HPDM method. In this case, the material and the size of the structure have no importance and they are inherited from the considered application. Indeed, the behaviour of the structure is the same as long as the orders of magnitude of the thickness to length ratios are the same, which implies that the dimensionless parameter $\lambda_{b} / \ell$ is unchanged. For example, we can replace concrete by a soft material and divide every length by 100 in Eq. (19). Then the characteristics of the 
structure become:

$$
\begin{array}{rlrl}
N & =15 & H & =N \ell_{w}=45 \mathrm{~cm} \\
\ell_{w} & =\ell_{f}=3 \mathrm{~cm} & h_{w} & =h_{f}=1 \mathrm{~cm} \\
a_{w} & =0.971 \mathrm{~cm} & a_{f} & =0.314 \mathrm{~cm} \\
E_{w} & =E_{f}=1 \mathrm{MPa} & \nu_{w} & =\nu_{f}=0.5 \\
\rho_{w} & =1000 \mathrm{~kg} \cdot \mathrm{m}^{-3} & \rho_{f} & =6185 \mathrm{~kg} \cdot \mathrm{m}^{-3}
\end{array}
$$

The density of the "floors" is still chosen in such a way that $\Lambda_{f} \approx \Lambda_{w}$. For this new structure, the frequency of the first bending mode of the floors is $45.6 \mathrm{~Hz}$. If the effects of the local resonance are ignored in the calculation of the frequencies of the longitudinal modes of the structure, Eq. (17) gives $f_{1}=12.4 \mathrm{~Hz}, f_{2}=37.3 \mathrm{~Hz}, f_{3}=62.1 \mathrm{~Hz}, f_{4}=87.0 \mathrm{~Hz}$. Since the resonance of the floors occurs in the same frequency range as the longitudinal modes, the behaviour of this structure is similar to the one of Section 4.3.

Moreover, it is possible to modify other parameters than the thickness to length ratio. The desired properties for a structure with an unconventional behaviour can be obtained by using different materials for the "walls" and the "floors" and by choosing different geometrical characteristics. An example is the choice of floors longer than the walls in Section 5.1. It is also possible to add point masses. If we impose $\rho_{f}=\rho_{w}=2300 \mathrm{~kg} . \mathrm{m}^{-3}, \ell_{f}=\ell_{w}=3 \mathrm{~m}$, $a_{f}=0.314 \mathrm{~m}$ and $a_{w}=0.971 \mathrm{~m}$ as in Section 4.3 , we should put a mass $m=11233.2 \mathrm{~kg}$ in the middle of the floors to have $\Lambda_{f}+m / \ell_{w}=\Lambda_{w}$. This structure has the same tension-compression stiffness and the same total mass as the example of Section 4.3 but the frequency of the resonance of the floors is lower $f_{f}=34 \mathrm{~Hz}$. However, it is still in the range of the frequencies of the longitudinal modes of the structure and the effects of the local resonance have to be taken into account. The expression of the effective mass has to be modified to include the inertia of the point masses. Moreover, the floors behave like spring-mass systems with one degree of freedom and one resonance. The attenuation of the vibrations of the structure at different frequencies can be achieved by using several types of resonating elements.

The results can also be extended to other geometries. For frame structures with a higher number of walls [Figure 16(a)], the generalization of Eq. (15) is straightforward: $2 E_{w} A_{w}$ and $\Lambda_{w}$ must be replaced by the sum of the tension-compression stiffness and the mass of all the walls; the contribution of the floors to the inertia forces is obtained by summing the effective mass calculated for each floor. If the structure has a more complex geom- 
etry, its macroscopic behaviour can be described by another equation but the physics remains the same. For example, the floors can be inclined as in Figure 16(b) to increase the bending stiffness of the structure. In this case, the tension-compression stiffness must include the stiffness of the inclined floors and the effective mass in the description of the longitudinal modes is determined by projecting the inertia forces onto the vertical direction. For such a structure, the local resonance in bending can also affect the transverse modes.
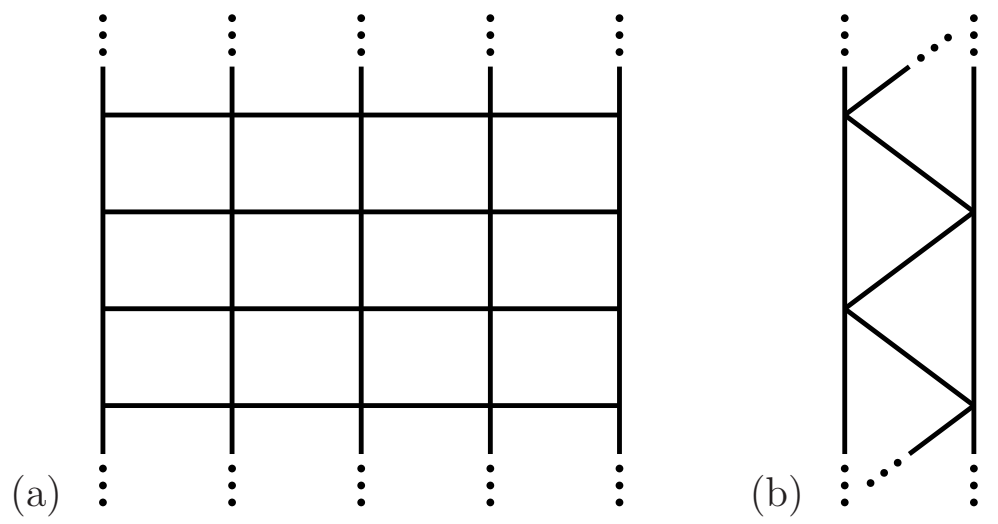

Figure 16: Other structures which can be affected by the local resonance in bending

\section{Conclusion}

Because of the stiffness contrast between tension-compression and bending in beams and plates, the longitudinal modes of a reticulated structure can appear in the same frequency range as the bending modes of the elements. The extension of the HPDM method to these situations with local resonance shows that the real mass should be replaced by an effective mass which depends on the frequency. When the frequency approaches the frequencies of the odd bending modes of the resonating elements, the effective mass becomes infinite and it changes its sign. Two consequences of this phenomenon have been highlighted. First, several normal modes of the structure associated with different natural frequencies can have the same macroscopic mode shape. Second, when the effective mass is negative, the vibrations are attenuated by the structure and we have frequency bandgaps.

These theoretical results are confirmed by finite element simulations made for two structures. The first one is an academic example specially designed 
to illustrate the effects of the local resonance. In particular, mass has been added to the floors. If the proportion of the mass affected by the resonance is smaller, the variations of the effective mass far from the resonance frequencies can be negligible. However, at the resonance, the effective mass is still considerable and the vibrations are attenuated. For the second structure, the dimensions of the elements were closer to the ones of the walls and the floors of real buildings. The effects of the local resonance are still visible but there are also modulated modes in the same frequency range. The design of these structures was made easier because the HPDM method is completely analytic. This provides a clear understanding of the mechanisms governing the behaviour of the structure and of the role of each mechanical or geometrical parameter.

This work focuses on the resonance of the floors because it is the most interesting situation [12]. Since the macroscopic description is given by the balance of the vertical forces, it cannot be affected by the bending resonance of the walls. In this case, only the inner equilibrium of the cell contains terms which depend on the frequency. The inertial terms become infinite at the frequencies of the odd bending modes of the walls but the bending stiffness becomes infinite at the frequencies of the even bending modes. When the bending resonance of the walls and the floors occurs in the same frequency range, the modes of the whole cell can also be excited. In the neighbourhood of all these frequencies, the structure is likely to behave atypically.

\section{A. Harmonic vibration of Euler-Bernoulli beams}
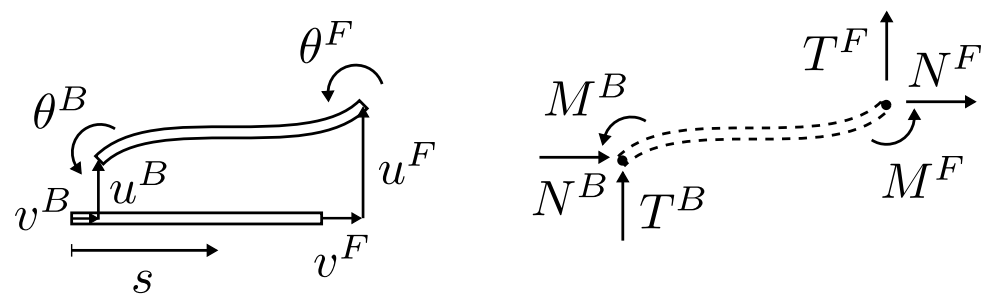

Figure 17: Studied beam

This appendix introduces some results about the harmonic vibrations of Euler-Bernoulli beams. We consider a beam of length $\ell$ linking the node $B$ to the node $F$ (see Figure 17). The same notation as in Section 2 is used for the characteristics of the beam. Moreover, in the local beam frame, $s$ 
stands for the coordinate along the beam axis, $u(s), v(s)$ for the transverse and axial displacements respectively, and $\theta(s)=u^{\prime}(s)$ for the rotation. The primes denote the differentiation with respect to $s$. The axial force $N(s)$, the shear force $T(s)$, and the bending moment $M(s)$ act by convention from the left part to the right part. No external force is applied on the beam.

\section{A.1. Tension-compression}

The longitudinal vibrations are described by the momentum balance along the beam axis and by the tension-compression constitutive law:

$$
\left\{\begin{aligned}
N^{\prime}(s) & =\rho A \omega^{2} v(s) \\
N(s) & =-E A v^{\prime}(s)
\end{aligned} \Rightarrow \quad \begin{array}{rl}
v^{\prime \prime}(s) & =-\chi^{2} v(s) \\
\text { where } \chi & =\sqrt{\frac{\rho \omega^{2}}{E}}=\frac{2 \pi}{\lambda_{c}}
\end{array}\right.
$$

$\lambda_{c}$ is the tension-compression wavelength in the beam at the studied circular frequency $\omega$. Therefore, the axial displacement can be written in the following way:

$$
v(s)=X \cos (\chi s)+Y \sin (\chi s)
$$

The two constants of integration $X$ and $Y$ are determined thanks to the boundary conditions.

\section{A.1.1. Expression of the nodal forces}

As explained in Appendix B, for the first step of the HPDM method, we need to express the forces at the extremities of the elements as functions of the nodal kinematic variables. The axial forces $N^{B}$ and $N^{F}$ in the local beam frame are obtained by using the unknown displacements $v^{B}$ and $v^{F}$ as boundary conditions:

$$
\begin{aligned}
& N^{B}=N\left(v^{B}, v^{F}\right) \quad \text { and } N^{F}=-N\left(v^{F}, v^{B}\right) \\
& \text { with } \quad N\left(v_{1}, v_{2}\right)=\frac{E A \chi}{\sin (\chi \ell)}\left(v_{1} \cos (\chi \ell)-v_{2}\right)
\end{aligned}
$$

At low frequencies, the beam has a quasi-static behaviour and the wavelength $\lambda_{c}$ is much longer than the length $\ell$ of the beam. Consequently, $\chi \ell$ is very small and the function $N\left(v_{1}, v_{2}\right)$ can be expanded:

$$
\begin{aligned}
N\left(v_{1}, v_{2}\right)=\frac{E A}{\ell}\left(\left(v_{1}-v_{2}\right)-\frac{(\chi \ell)^{2}}{6}\right. & \left(2 v_{1}+v_{2}\right) \\
- & \left.\frac{(\chi \ell)^{4}}{360}\left(8 v_{1}+7 v_{2}\right)\right)+O\left((\chi \ell)^{6}\right)
\end{aligned}
$$




\section{A.1.2. Natural frequencies of the walls}

We are also interested in the natural frequencies of the walls considered as clamped-free beams. In that case, the boundary conditions are $v(0)=0$ and $N(\ell)=0$. The circular frequency of the $k$ th mode is given by the following equation:

$$
\cos (\chi \ell)=0 \quad \Rightarrow \quad \omega_{k}=(2 k-1) \frac{\pi}{2 \ell} \sqrt{\frac{E}{\rho}}
$$

\section{A.2. Bending}

The transverse vibrations are described by the momentum balance along the transverse axis, the moment of momentum balance, and the bending constitutive law:

$$
\left\{\begin{aligned}
T^{\prime}(s) & =\rho A \omega^{2} u(s) \\
M^{\prime}(s) & =-T(s) \\
M(s) & =-E I u^{\prime \prime}(s)
\end{aligned} \quad \Rightarrow \quad \begin{array}{rl}
u^{\prime \prime \prime \prime}(s) & =\beta^{4} u(s) \\
& \text { where } \beta=\sqrt[4]{\frac{\rho A \omega^{2}}{E I}}=\frac{2 \pi}{\lambda_{b}}
\end{array}\right.
$$

$\lambda_{b}$ is the bending wavelength in the beam at the studied circular frequency $\omega$. Since the Euler-Bernoulli beam description requires that the bending wavelength is much greater than the thickness of the beam, the bending wavelength is always smaller than the tension-compression wavelength:

$$
\lambda_{b}^{2}=\lambda_{c} 2 \pi \sqrt{\frac{I}{A}}=\lambda_{c} \frac{2 \pi a}{\sqrt{12}} \quad \Rightarrow \quad \frac{\lambda_{b}}{\lambda_{c}}=O\left(\frac{a}{\lambda_{b}}\right)<<1
$$

Eq. (28) shows that the transverse displacement can be written in the following way:

$$
u(s)=X \cos (\beta s)+Y \sin (\beta s)+Z \cosh (\beta s)+W \sinh (\beta s)
$$

The four constants of integration $X, Y, Z$ and $W$ are determined thanks to the boundary conditions.

\section{A.2.1. Expression of the nodal forces and moments}

We first use the unknown displacements $u^{B}, u^{F}$ and rotations $\theta^{B}, \theta^{F}$ as boundary conditions to obtain the expressions of the shear forces $T^{B}, T^{F}$ and the bending moments $M^{B}, M^{F}$ at the extremities of the beam in the local frame.

$$
\begin{aligned}
T^{B} & =T\left(u^{B}, u^{F}, \theta^{B}, \theta^{F}\right) \quad ; \quad T^{F} & =T\left(-u^{F},-u^{B}, \theta^{F}, \theta^{B}\right) \\
M^{B} & =M\left(u^{B}, u^{F}, \theta^{B}, \theta^{F}\right) \quad ; \quad & M^{F}=M\left(u^{F}, u^{B},-\theta^{F},-\theta^{B}\right)
\end{aligned}
$$


with

$$
\begin{aligned}
& T\left(u_{1}, u_{2}, \theta_{1}, \theta_{2}\right)=\frac{E I \beta^{3}}{1-\cos (\beta \ell) \cosh (\beta \ell)}\left(u_{1}[\cosh (\beta \ell) \sin (\beta \ell)+\sinh (\beta \ell) \cos (\beta \ell)]\right. \\
& \left.-u_{2}[\sin (\beta \ell)+\sinh (\beta \ell)]+\frac{\theta_{1}}{\beta} \sin (\beta \ell) \sinh (\beta \ell)-\frac{\theta_{2}}{\beta}[\cos (\beta \ell)-\cosh (\beta \ell)]\right) \\
& M\left(u_{1}, u_{2}, \theta_{1}, \theta_{2}\right)=\frac{E I \beta^{2}}{1-\cos (\beta \ell) \cosh (\beta \ell)}\left(u_{1} \sin (\beta \ell) \sinh (\beta \ell)\right. \\
& +u_{2}[\cos (\beta \ell)-\cosh (\beta \ell)]+\frac{\theta_{1}}{\beta}[\cosh (\beta \ell) \sin (\beta \ell)-\sinh (\beta \ell) \cos (\beta \ell)] \\
& \left.-\frac{\theta_{2}}{\beta}[\sin (\beta \ell)-\sinh (\beta \ell)]\right)
\end{aligned}
$$

At low frequencies, when the wavelength $\lambda_{b}$ is much longer than the length $\ell$ of the beam, $\beta \ell$ is very small. The shear forces and the bending moments can be expanded according to the powers of $\beta \ell$.

$$
\begin{aligned}
& T\left(u_{1}, u_{2}, \theta_{1}, \theta_{2}\right)=-\frac{12 E I}{\ell^{3}}\left(\left(u_{1}-u_{2}\right)+\frac{\ell}{2}\left(\theta_{1}+\theta_{2}\right)\right. \\
& \left.-\frac{(\beta \ell)^{4}}{840}\left(26 u_{1}+9 u_{2}\right)-\frac{(\beta \ell)^{4} \ell}{5040}\left(22 \theta_{1}-13 \theta_{2}\right)\right)+O\left((\beta \ell)^{8}\right) \\
& M\left(u_{1}, u_{2}, \theta_{1}, \theta_{2}\right)=\frac{6 E I}{\ell^{2}}\left(\left(u_{1}-u_{2}\right)+\frac{\ell}{3}\left(2 \theta_{1}+\theta_{2}\right)\right. \\
& \left.-\frac{(\beta \ell)^{4}}{2520}\left(22 u_{1}+13 u_{2}\right)-\frac{(\beta \ell)^{4} \ell}{2520}\left(4 \theta_{1}-3 \theta_{2}\right)\right)+O\left((\beta \ell)^{8}\right)
\end{aligned}
$$

\section{A.2.2. Natural frequencies of a clamped beam}

We now introduce some properties of the natural frequencies of a clampedclamped beam which are useful for the interpretation of the results about the local resonance. The boundary conditions are:

$$
\begin{aligned}
& u(0)=X+Z=0 \\
& \theta(0)=\beta(Y+W)=0 \\
& u(\ell)=X \cos (\beta \ell)+Y \sin (\beta \ell)+Z \cosh (\beta \ell)+W \sinh (\beta \ell)=0 \\
& \theta(\ell)=\beta(-X \sin (\beta \ell)+Y \cos (\beta \ell)+Z \sinh (\beta \ell)+W \cosh (\beta \ell))=0
\end{aligned}
$$

Eqs. (34a) and (34b) are used to eliminate $Z$ and $W$ in Eqs. (34c) and (34d). The existence of a non-zero solution implies that the determinant 
of Eqs. (34c) and (34d) is zero. This leads to the following equation the solutions of which are the natural frequencies of the clamped-clamped beam:

$$
1-\cos (\beta \ell) \cosh (\beta \ell)=0
$$

This equation can also be written in the following way:

$$
\begin{gathered}
2 f_{o}(\beta \ell) f_{e}(\beta \ell)=0 \\
\text { with } \quad \begin{aligned}
& f_{o}(\beta \ell)= \sin \left(\frac{\beta \ell}{2}\right) \cosh \left(\frac{\beta \ell}{2}\right)+\sinh \left(\frac{\beta \ell}{2}\right) \cos \left(\frac{\beta \ell}{2}\right) \\
& f_{e}(\beta \ell)=\sin \left(\frac{\beta \ell}{2}\right) \cosh \left(\frac{\beta \ell}{2}\right)-\sinh \left(\frac{\beta \ell}{2}\right) \cos \left(\frac{\beta \ell}{2}\right)
\end{aligned}
\end{gathered}
$$

The function $f_{o}(\beta \ell)$ vanishes at the frequencies of the odd modes and the function $f_{e}(\beta \ell)$ vanishes at the frequencies of the even modes.

The first solution of Eq. (35) is $\beta \ell \approx 4.73$ which is close to $3 \pi / 2 \approx 4.71$. As $\cosh (\beta \ell)$ becomes very important for the higher values of $\beta \ell$, the solutions of $\cos (\beta \ell)=0$ approximate very well the other solutions of Eq. (35). Therefore, the solutions of Eq. (35) are close to $(2 k+1) \pi / 2$ and the natural frequencies of a clamped-clamped beam are almost proportional to the sequence of the squares of the odd integers.

$$
(\beta \ell)_{k} \approx(2 k+1) \frac{\pi}{2} \quad \Rightarrow \quad \omega_{k} \approx(2 k+1)^{2}\left(\frac{\pi}{2 \ell}\right)^{2} \sqrt{\frac{E I}{\rho A}}
$$

Note that the parameter $\beta$ can be written as a function of the dimensionless frequency $\omega / \omega_{1}$ :

$$
\beta \approx \frac{3 \pi}{2 \ell} \sqrt{\frac{\omega}{\omega_{1}}}
$$

\section{A.2.3. Deformation of the floors}

Finally, we examine the deformation of the floors when the structure experiences longitudinal vibrations. In that case, the beam is excited by an identical transverse motion and an opposite rotation of the nodes. The boundary conditions are:

$$
u(0)=u(\ell)=V \quad ; \quad \theta(0)=-\theta(\ell)=\frac{\Phi}{2}
$$


This leads to the following expression of the transverse displacement:

$$
\begin{aligned}
u(s)=\frac{\sinh \left(\frac{\beta \ell}{2}\right) \sin \left(\frac{\beta \ell}{2}\right)}{f_{o}(\beta \ell)}([\sin (\beta x)-\sinh (\beta x) \\
\left.+\operatorname{coth}\left(\frac{\beta \ell}{2}\right) \cosh (\beta x)+\cot \left(\frac{\beta \ell}{2}\right) \cos (\beta x)\right] V \\
+\left[\operatorname{coth}\left(\frac{\beta \ell}{2}\right) \sin (\beta x)+\cot \left(\frac{\beta \ell}{2}\right) \sinh (\beta x)\right. \\
\left.\left.+\operatorname{coth}\left(\frac{\beta \ell}{2}\right) \cot \left(\frac{\beta \ell}{2}\right)[\cos (\beta x)-\cosh (\beta x)]\right] \frac{\Phi}{2 \beta}\right)
\end{aligned}
$$

The sum of the inertia forces acting on the beam is then:

$$
\int_{0}^{\ell} \rho \omega^{2} u(s) \mathrm{d} s=\frac{\rho \omega^{2}}{\beta f_{o}(\beta \ell)}\left[4 \sin \left(\frac{\beta \ell}{2}\right) \sinh \left(\frac{\beta \ell}{2}\right) V+f_{e}(\beta \ell) \frac{\Phi}{\beta}\right]
$$

If $\beta$ is replaced by the expression (38), it becomes:

$$
\begin{aligned}
\int_{0}^{\ell} \rho \omega^{2} u(s) \mathrm{d} s & =\frac{2 \ell \rho \omega^{2}}{3 \pi \sqrt{\frac{\omega}{\omega_{1}}} f_{o}\left(\frac{3 \pi}{2} \sqrt{\frac{\omega}{\omega_{1}}}\right)} \\
& {\left[4 \sin \left(\frac{3 \pi}{4} \sqrt{\frac{\omega}{\omega_{1}}}\right) \sinh \left(\frac{3 \pi}{4} \sqrt{\frac{\omega}{\omega_{1}}}\right) V+f_{e}\left(\frac{3 \pi}{2} \sqrt{\frac{\omega}{\omega_{1}}}\right) \frac{2 \ell \Phi}{3 \pi \sqrt{\frac{\omega}{\omega_{1}}}}\right] }
\end{aligned}
$$

\section{B. Homogenization method of periodic discrete media}

This appendix describes the implementation of the HPDM method in the case of the frame structures of Figure 1. A more general presentation of the method can be found in [22] and other examples of application are given in $[23,24,25,26,27]$. The adaptation of the method to situations with local resonance is discussed in [7]. The method includes two main steps: the discretization of the dynamic balance of the structure followed by the homogenization process. The notation is the same as in Section 2 and Appendix A.

The aim of the first step is to concentrate on the nodes the study of the momentum balance without loss of information. The frame structure is 
considered as a periodic lattice of interconnected beams. The balances of the elements in tension-compression and in bending are written as explained in Appendix A. This provides the expressions of the forces and moments at the extremities of the elements in their local frame [Eqs. (25), (31) and (32)].

The dynamic balance of each element being satisfied, it remains to introduce the geometry of the structure by writing the balance of the nodes. Because their mass is negligible and there is no external force, it consists in adding the forces or moments applied by the two walls and the floor connected to the same node. Moreover, the connection is perfectly stiff and the motions of the three elements are identical. Therefore, the nodal balance equations are a set of finite difference equations expressed in the global frame $\left(\mathbf{e}_{1}, \mathbf{e}_{2}, \mathbf{e}_{3}\right)$ of Figure 1 and depending only on the nodal kinematic variables defined in Section 2.1. For each level $n$, there are three balance equations for the node $n_{1}$ and three balance equations for the node $n_{2}$.

Balance of the forces in the horizontal direction $\left(\mathbf{e}_{2}\right)$ :

$$
\begin{array}{r}
\text { Node } n_{1} \\
T_{w}^{F}\left(u_{(n-1)_{1}}, u_{n_{1}}, \theta_{(n-1)_{1}}, \theta_{n_{1}}\right)-T_{w}^{B}\left(u_{n_{1}}, u_{(n+1)_{1}}, \theta_{n_{1}}, \theta_{(n+1)_{1}}\right) \\
-N_{f}^{B}\left(u_{n_{1}}, u_{n_{2}}\right)=0
\end{array}
$$

Balance of the forces in the vertical direction $\left(\mathbf{e}_{1}\right)$ :

$$
\begin{gathered}
\text { Node } n_{1} \\
N_{w}^{F}\left(v_{(n-1)_{1}}, v_{n_{1}}\right)-N_{w}^{B}\left(v_{n_{1}}, v_{(n+1)_{1}}\right)-T_{f}^{B}\left(v_{n_{1}}, v_{n_{2}},-\theta_{n_{1}},-\theta_{n_{2}}\right)=0 \\
\text { Node } n_{2} \\
N_{w}^{F}\left(v_{(n-1)_{2}}, v_{n_{2}}\right)-N_{w}^{B}\left(v_{n_{2}}, v_{(n+1)_{2}}\right)+T_{f}^{F}\left(v_{n_{1}}, v_{n_{2}},-\theta_{n_{1}},-\theta_{n_{2}}\right)=0
\end{gathered}
$$


Balance of the moments:

$$
\begin{gathered}
\text { Node } n_{1} \\
M_{w}^{F}\left(u_{(n-1)_{1}}, u_{n_{1}}, \theta_{(n-1)_{1}}, \theta_{n_{1}}\right)- \\
+M_{w}^{B}\left(u_{n_{1}}, u_{(n+1)_{1}}, \theta_{n_{1}}, \theta_{(n+1)_{1}}\right) \\
+M_{f}^{B}\left(v_{n_{1}}, v_{n_{2}},-\theta_{n_{1}},-\theta_{n_{2}}\right)=0 \\
\text { Node } n_{2} \\
M_{w}^{F}\left(u_{(n-1)_{2}}, u_{n_{2}}, \theta_{(n-1)_{2}}, \theta_{n_{2}}\right)-M_{w}^{B}\left(u_{n_{2}}, u_{(n+1)_{2}}, \theta_{n_{2}}, \theta_{(n+1)_{2}}\right) \\
-M_{f}^{F}\left(v_{n_{1}}, v_{n_{2}},-\theta_{n_{1}},-\theta_{n_{2}}\right)=0
\end{gathered}
$$

The use of the change of variables proposed in Section 2.1 [Eqs. (1) and (2)] and the combination of the balance equations in the following way provides two uncoupled sets of three equations. The first one [Eqs. (ST), (DN) and $(\mathrm{SM})$ ] describes the transverse kinematics and the second one [Eqs. (DT), $(\mathrm{SN})$ and $(\mathrm{DM})$ ] describes the longitudinal kinematics.

$$
\begin{gathered}
(\mathrm{ST})=\frac{(43 \mathrm{a})+(43 \mathrm{~b})}{2} \\
T_{w}^{F}\left(U_{n-1}, U_{n}, \theta_{n-1}, \theta_{n}\right)-T_{w}^{B}\left(U_{n}, U_{n+1}, \theta_{n}, \theta_{n+1}\right)-N_{f}^{B}\left(U_{n}, U_{n}\right)=0 \\
(\mathrm{DN})=\frac{(43 \mathrm{~d})-(43 \mathrm{c})}{2} \\
-\frac{\ell_{f}}{2} N_{w}^{F}\left(\alpha_{n-1}, \alpha_{n}\right)+\frac{\ell_{f}}{2} N_{w}^{B}\left(\alpha_{n}, \alpha_{n+1}\right)-T_{f}^{B}\left(-\frac{\ell_{f}}{2} \alpha_{n}, \frac{\ell_{f}}{2} \alpha_{n}, \theta_{n}, \theta_{n}\right)=0 \\
(\mathrm{SM})=\frac{(43 \mathrm{e})+(43 \mathrm{f})}{2} \\
M_{w}^{F}\left(U_{n-1}, U_{n}, \theta_{n-1}, \theta_{n}\right)-M_{w}^{B}\left(U_{n}, U_{n+1}, \theta_{n}, \theta_{n+1}\right) \\
-M_{f}^{B}\left(-\frac{\ell_{f}}{2} \alpha_{n}, \frac{\ell_{f}}{2} \alpha_{n}, \theta_{n}, \theta_{n}\right)=0 \\
(\mathrm{DT})=\frac{(43 \mathrm{~b})-(43 \mathrm{a})}{2} \\
\frac{1}{2} T_{w}^{B}\left(\Delta_{n}, \Delta_{n+1}, \Phi_{n}, \Phi_{n+1}\right)-\frac{1}{2} N_{f}^{B}\left(\Delta_{n},-\Delta_{n}\right)=0
\end{gathered}
$$




$$
\begin{gathered}
(\mathrm{SN})=\frac{(43 \mathrm{c})+(43 \mathrm{~d})}{2} \\
N_{w}^{F}\left(V_{n-1}, V_{n}\right)-N_{w}^{B}\left(V_{n}, V_{n+1}\right)-T_{f}^{B}\left(V_{n}, V_{n}, \frac{\Phi_{n}}{2},-\frac{\Phi_{n}}{2}\right)=0 \\
(\mathrm{DM})=\frac{(43 \mathrm{f})-(43 \mathrm{e})}{2} \\
\frac{1}{2} M_{w}^{F}\left(\Delta_{n-1}, \Delta_{n}, \Phi_{n-1}, \Phi_{n}\right)-\frac{1}{2} M_{w}^{B}\left(\Delta_{n}, \Delta_{n+1}, \Phi_{n}, \Phi_{n+1}\right) \\
-M_{f}^{B}\left(V_{n}, V_{n}, \frac{\Phi_{n}}{2},-\frac{\Phi_{n}}{2}\right)=0
\end{gathered}
$$

Once these equations are solved and the nodal kinematic variables are determined, the motions $u, v, \theta$, the forces $N, T$ and the moment $M$ inside each element can always be calculated thanks to the equations of Appendix A. The discrete description constituted by Eqs. (44), (1) and (2) is a complete description of the structure which contains all the necessary information. It is thus possible to use these variables as descriptors of the motion of the structure.

Then the principles of homogenization are used to turn the discrete description into a continuous description valid at the macroscopic scale. The aim is to obtain the differential equation describing the behaviour of the equivalent beam. The key assumption is scale separation. This means that the cell size in the direction of periodicity $\ell_{w}$ is small compared to the unknown characteristic size $L$ of the vibrations of the structure. Consequently, the scale ratio $\epsilon=\ell_{w} / L$ is a small parameter $(\epsilon<<1)$ used for the expansion of the unknowns and the forces.

The classical homogenization considers frequencies much smaller than the natural frequencies of the frame elements. In that case, the condition of scale separation is always respected. The tension-compression wavelength $\lambda_{c}$ and the bending wavelength $\lambda_{b}$ in the elements are much longer than their length $\ell$. It is therefore possible to expand the nodal forces and moment according to the small parameters $\chi \ell$ and $\beta \ell$ as in Eqs. (26) and (33) of Appendix A. However, having a quasi-static state at the local scale is only a sufficient condition and homogenization can be extended to situations with local resonance [7]. As $\lambda_{b}$ is always smaller than $\lambda_{c}$ in beams, there is a frequency range with $\lambda_{b}=O(\ell)$ and $\lambda_{c}>>\ell$. The corresponding elements are in resonance in bending and the expansion of the shear force and the 
bending moment is no longer possible. But we can expand the axial force and apply homogenization. This method is also valid at higher frequencies [with $\lambda_{b}<O(\ell)$ ] provided that $\lambda_{c}$ remains sufficiently long to define a macroscopic scale and that the behaviour of the elements is correctly described by the beam model.

Another consequence of scale separation is that the nodal motions slowly vary from one level to the next. The kinematic descriptors can therefore be considered as the discrete values of continuous functions of the macroscopic space variable $x$. These functions are assumed to converge as $\epsilon$ approaches zero and are replaced by asymptotic expansions in powers of $\epsilon$ [Eq. (3) or (9)]. Equations (44) describing the balance of a level also depend on the motions of the two neighbouring levels. The distance between the levels is constant because of the periodicity of the structure and is equal to $\ell_{w}=\epsilon L$. This value is small with respect to $x$, which enables the variations of the motions to be expressed with Taylor's series. This introduces the macroscopic derivatives.

$$
X_{n \pm 1}=X^{0}\left(n \ell_{w}\right)+\epsilon\left(X^{1}\left(n \ell_{w}\right) \pm L \frac{\partial X^{0}}{\partial x}\left(n \ell_{w}\right)\right)+\ldots
$$

where $X$ stands for one of the kinematic descriptors.

To correctly take into account the local physics, the physical parameters have to be scaled according to the powers of $\epsilon$. This normalization ensures that each mechanical effect appears at the same order whatever the value of $\epsilon$. Thus, the same physics is kept when $\epsilon$ approaches zero, i.e. in the homogenized model. The choice of the properties of the elements determines the stiffness contrast between the various mechanisms and then the nature of the equivalent beam. The order of magnitude of the frequency is imposed by the balance of the elastic and inertia forces at the macroscopic scale. For the study of the longitudinal modes, the inertia forces must have the same order of magnitude as the tension-compression forces in the walls. Note that the natural frequencies of the structure are unknown and must therefore be written in the form of asymptotic expansions:

$$
\omega=\epsilon^{m}\left(\omega_{0}+\epsilon \omega_{1}+\epsilon^{2} \omega_{2}+\ldots\right)=\tilde{\omega}_{0}+\tilde{\omega}_{1}+\tilde{\omega}_{2}+\ldots
$$

where $\epsilon^{m}$ is the order of magnitude of the (circular) frequency.

Finally, all the expansions in powers of $\epsilon$ and the normalization are introduced in the balance equations. These relations are valid for any small enough $\epsilon$ and the orders can be separated. This leads for each $\epsilon$ order to a 
set of differential equations, which can be solved in increasing order. The homogenized model is given by the leading order, that is to say the limit when $\epsilon$ approaches 0 . The higher orders are correctors which improve the macroscopic description when the scale separation is poor.

\section{Acknowledgements}

The authors thank B. Peboscq for doing some of the calculations.

\section{References}

[1] J-L. Auriault, G. Bonnet, Dynamique des composites élastiques, Arch. Mech. 37 (4-5) (1985) 269-284.

[2] J-L. Auriault, C. Boutin, Long wavelength inner-resonance cut-off frequencies in elastic composite materials, Int. J. Solids Struct. 49 (23-24) (2012) 3269-3281. doi:10.1016/j.ijsolstr.2012.07.002.

[3] Z. Liu, X. X. Zhang, Y. W. Mao, Y. Y. Zhu, Z. Y. Yang, C. T. Chan, P. Sheng, Locally resonant sonic materials, Sci. 289 (5485) (2000) 17341736. doi:10.1126/science.289.5485.1734.

[4] Y. Shanshan, Z. Xiaoming, H. Gengkai, Experimental study on negative effective mass in a 1D mass-spring system, New J. Phys. 10 (043020) (2008) 1-11. doi:10.1088/1367-2630/10/4/043020.

[5] G. A. Maugin, On some generalizations of Boussinesq and KdV Systems, Proc. Estonian Acad. Sci. Phys. Math. 44 (1) (1995) 40-55.

[6] C. Chesnais, S. Hans, C. Boutin, Wave propagation and diffraction in discrete structures: Effect of anisotropy and internal resonance, PAMM 7 (1) (2007) 1090401-1090402.

[7] C. Chesnais, C. Boutin, S. Hans, Effects of the local resonance on the wave propagation in periodic frame structures: Generalized Newtonian mechanics, J. Acoust. Soc. Am. 132 (4, Part 2) (2012) 2873-2886. doi : 10.1121/1.4744975.

[8] E. Baravelli, M. Ruzzene, Internally resonating lattices for bandgap generation and low-frequency vibration control, J. Sound Vib. 332 (25) (2013) 6562-6579. doi:10.1016/j.jsv.2013.08.014. 
[9] D. Caillerie, P. Trompette, P. Verna, Homogenisation of periodic trusses, in: IASS Symposium, 10 Years of Progress in Shell and Spatial Structures, Madrid, 1989.

[10] C. Boutin, S. Hans, Homogenisation of periodic discrete medium: Application to dynamics of framed structures, Comput. Geotech. 30 (4) (2003) 303-320. doi:10.1016/S0266-352X (03)00005-3.

[11] S. Hans, C. Boutin, Dynamics of discrete framed structures: A unified homogenized description, J. Mech. Mater. Struct. 3 (9) (2008) 17091739. doi:10.2140/jomms.2008.3.1709.

[12] C. Chesnais, Dynamique de milieux réticulés non contreventés - Application aux bâtiments (Dynamics of unbraced reticulated media - Application to buildings), Ph.D. thesis, ENTPE - ECL, (accessed 17 February 2015) (2010).

URL http://bibli.ec-lyon.fr/exl-doc/TH_T2177_cchesnais.pdf

[13] O. A. B. Hassan, New methods for reducing ground-borne noise in buildings above railway tunnels, Ph.D. thesis, Kungl Tekniska Högskolan, Stockholm (2003).

[14] L. Auersch, Building response due to ground vibration - Simple prediction model based on experience with detailed models and measurements, Int. J. Acoust. Vib. 15 (3) (2010) 101-112.

[15] A. J. Papazoglou, A. S. Elnashai, Analytical and field evidence of the damaging effect of vertical earthquake ground motion, Earthq. Eng. Struct. Dyn. 25 (10) (1996) 1109-1137. doi : 10.1002/(SICI) 1096-9845(199610) $25: 1$.

[16] I. L. Manevitch, G. V. Oshmyan, An asymptotic study of the linear vibrations of a stretched beam with concentrated masses and discrete elastic supports, J. Sound Vib. 223 (5) (1999) 679-691. doi:10.1006/jsvi.1998.1889.

[17] E. M. Daya, B. Braikat, N. Damil, M. Potier-Ferry, Continuum modeling for the modulated vibration modes of large repetitive structures, C. R. Mec. 330 (5) (2002) 333-338. doi:10.1016/S1631-0721(02)01464-X. 
[18] N. Moustaghfir, E. M. Daya, B. Braikat, N. Damil, M. Potier-Ferry, Evaluation of continuous modelings for the modulated vibration modes of long repetitive structures, Int. J. Solids Struct. 44 (21) (2007) 70617072. doi:10.1016/j.ijsolstr.2007.03.023.

[19] E. Nolde, R. V. Craster, J. Kaplunov, High frequency homogenization for structural mechanics, J. Mech. Phys. Solids 59 (3) (2011) 651-671. doi:10.1016/j.jmps.2010.12.004.

[20] R. V. Craster, L. M. Joseph, J. Kaplunov, Long-wave asymptotic theories: The connection between functionally graded waveguides and periodic media, Wave Motion 51 (4) (2014) 581-588. doi:10.1016/j. wavemoti.2013.09.007.

[21] C. Boutin, A. Rallu, S. Hans, Large scale modulation of high frequency waves in periodic elastic composites, J. Mech. Phys. Solids 70 (2014) 362381. doi:10.1016/j.jmps.2014.05.015.

[22] H. Tollenaere, D. Caillerie, Continuous modeling of lattice structures by homogenization, Adv. Eng. Softw. 29 (7-9) (1998) 699-705. doi:10.1016/S0965-9978(98)00034-9.

[23] G. Moreau, D. Caillerie, Continuum modeling of lattice structures in large displacement applications to buckling analysis, Comput. Struct. 68 (1-3) (1998) 181-189. doi:10.1016/S0045-7949(98)00041-8.

[24] D. Caillerie, A. Mourad, A. Raoult, Cell-to-muscle homogenization. Application to a constitutive law for the myocardium, ESAIM-Math. Model. Numer. Anal. 37 (4) (2003) 681-698. doi:10.1051/m2an: 2003054 .

[25] D. Caillerie, A. Mourad, A. Raoult, Discrete homogenization in graphene sheet modeling, J. Elast. 84 (1) (2006) 33-68. doi:10.1007/s10659-006-9053-5.

[26] C. Chesnais, S. Hans, C. Boutin, Dynamics of reticulated structures. Evidence of atypical gyration modes., Int. J. Multiscale Comput. Eng. 9 (5) (2011) 515-528. doi:10.1615/Int JMultCompEng. 2011002351. 
[27] C. Chesnais, C. Boutin, S. Hans, Wave propagation and non-local effects in periodic frame materials: Generalized continuum mechanics, Math. Mech. Solids (Published online before print, 2013) 1-30. doi: 10.1177/1081286513511092. 